\title{
Synthesis of thiophenylazolyl pyrrolylsulfamoyl acetamides as potential antimicrobial agents
}

\section{Tankam Rajeswari}

Sri Venkateswara University

\section{Panga Siva Sankar}

Sri Venkateswara University

\section{Kayathi Narendra Babu}

Sri Venkateswara University

\section{Adivireddy Padmaja}

Sri Venkateswara University

Venkatapuram Padmavathi ( $\nabla$ vkpuram2001@yahoo.com )

Sri Venkateswara University https://orcid.org/0000-0003-3448-1423

\section{Research Article}

Keywords: Azoles, Sulfamoyl acetamides, Antimicrobial activity

Posted Date: September 28th, 2021

DOI: https://doi.org/10.21203/rs.3.rs-921900/v1

License: (c) (i) This work is licensed under a Creative Commons Attribution 4.0 International License. Read Full License 


\section{Abstract}

A variety of thiophenylazolyl pyrrolylsulfamoyl acetamides were prepared by the reaction of azolylsulfamoyl acetate with pyrrolylamine in the presence of sodium methoxide in methanol under ultrasonication. Chloro, nitro and dinitro substituted thiophenylthiazolyl pyrrolylsulfamoyl acetamides (9d, 9e, 9f) and dinitro substituted thiophenylimidazolyl pyrrolylsulfamoyl acetamide (10f) exhibited potential antibacterial activity against $B$. subtilis. The compound $\mathbf{9 f}$ also displayed prominent antibacterial activity against $S$. aureus. The compounds 9 f, chloro and nitro substituted thiophenylimidazolyl pyrrolylsulfamoyl acetamide (10d, 10e) and 10f exhibited prominent antifungal activity against A.niger.

\section{Introduction}

Azoles are the most prevalent heteroaromatic molecules and display a pivotal role in drug discovery. Oxazole containing molecules viz., Inthomycin C (antineoplastic) [Ibrahim et al. 2017; K. J. Hale et al. 2014], Oxaprozin (anti-inflammatory) [Mark et al. 2011], Pemoline (nervous system stimulant) [Erhorn et al. 2007, Kulikowska et al. 2002] and Bengazole A (antibiotic) [Chandrasekhar et al. 2010] are used as drugs. Thiazole derivatives are common entities in pharmaceutical arena due to their broad applications in drug development [Valentin et al. 2010; Ahmed et al. 2007; Wilson et al. 2001; Ritesh et al. 2009]. Thiazole nucleus is also an integral part of penicillins, which have revolutionized the therapy of bacterial diseases [Samir et al. 2013]. Sulfathiazole (antimicrobial), Bleomycin and Tiazofurin (antineoplastic) are some of the drugs having thiazole moiety. Imidazoles form the main structure of some well-known biomolecules of human organisms such as Histidine, Vit B12, Histamine and Biotin [Leyla et al. 2013]. Pyrrole is another important scaffold found in complex macromolecules- porphyrins of heme, chlorins, bacteriochlorins, chlorophyll and porphyrinogens [Ramandeep et al. 2018; Andrea et al. 2002; Huihua et al. 2018; Honey et al. 2012]. Various pyrrole derivatives find application as antibacterial, [Roland et al. 2004] antiviral, [Kristina et al. 2007] anti-inflammatory [Harrak et al. 2007], antifungal [Ming et al. 2011], antioxidant [Jacques et al. 1999] and anticancer agents [Debasish et al. 2012]. Besides thiophene containing molecules are known to exhibit antimicrobial [Samir et al. 2010], anti-inflammatory [Przybilla et al. 1987], platelet inhibitory [John et al. 2012], antitumor [Ronald et al. 2002] and antiviral activities [Hudson et al. 1986]. The structure-activity relationship of the molecules revealed that change of substituents resulted in varied biopotency. Our continued interest towards the development of potential heteroaromatics [Siva Sankar et al. 2019; Sowmya et al. 2018; Rekha et al. 2017], we herein report the design and synthesis of thiophenylazolyl pyrrolylsulfamoyl acetamides as potential antimicrobial agents.

\section{Results And Discussion}

\section{Chemistry}

The azolyl amines- 4-thiophenyloxazolyl-2-amine (1) and 4-thiophenylthiazolyl-2-amine (2) were prepared by the cyclocondensation of 2-bromo-1-(thiophen-2-yl)ethan-1-one with urea and thiourea in methanol [Taterao et al. 2008]. 4-Thiophenylimidazolyl-2-amine (3) was prepared by the hydrolysis of $\mathrm{N}$-4-thiophenyl- $1 \mathrm{H}$-imidazol-2ylacetamide in the presence of $\mathrm{H}_{2} \mathrm{SO}_{4}$. The latter compound was obtained by the treatment of 2-bromo-1(thiophen-2-yl)ethan-1-one with acetylguanidine [Thomas et al. 1994]. Sulfonylation of heteroarylamines was reported by several methods [Divya et al. 2015; Pasha et al. 2014]. In fact, we have carried out $N$-sulfonylation of heteroarylamines with ethyl chlorosulfonylacetate in the presence of DMAP / $\mathrm{Et}_{3} \mathrm{~N}$ [Premakumari et al. 2014] and 
also in the presence of dispersed sodium in THF [Divya et al. 2015]. It was observed that the reaction proceeded at a faster rate with dispersed $\mathrm{Na}$. Based on these results, we have carried out the reaction of (1) with methyl 2(chlorosulfonyl) acetate in the presence of dispersed sodium under ultrasonication at a frequency of $35 \mathrm{KHz}$ where in the product methyl 2-(N-(4-(4-thiophen-2-yl)oxazol-2-yl)sulfamoyl)acetate (4) was obtained in excellent yield. Adopting similar methodology, methyl 2-(N-(4-(4-thiophen-2-yl)thiazol-2-yl)sulfamoyl)acetate (5) and methyl 2-(N(4-(4-thiophen-2-yl)-1 H-imidazol-2-yl)sulfamoyl)acetate (6) were prepared by the treatment of $\mathbf{2}$ and $\mathbf{3}$ with methyl 2-(chlorosulfonyl)acetate (Scheme I). In the presence of dispersed sodium, methyl 2-sulfonylacetate free radical was generated from methyl 2-chlorosulfonylacetate which reacted with heteroarylamines to get corresponding sulfonamides by the expulsion of $\mathrm{H}_{2}$ (Scheme I \& Mechanism).

\section{Mechanism}

Amidation of esters with amines was reported in the presence of different catalysts viz, DBU [Marcocci et al. 1994] triazabicyclo[4.4.0]dec-5-ene [Ahmad et al. 2011] and 1,2,4-triazole-DBU [Zia-ur-Rehman et al. 2009]. Inorganic catalysts like $\mathrm{Sb}(\mathrm{OEt})_{3}$ [Kristin et al. 2009] and $\mathrm{Zr}(\mathrm{OtBu})_{4}$-HoAt [Sabot et al. 2007] have also been used for direct amidation. Apart from these Takastri et al reported sodium methoxide catalyzed amidation of alkyl and aryl esters and proved sodium / potassium alkoxides possess high catalytic activity for direct amidation reactions [Xing et al. 2009]. As such we have carried out amidation of methyl 2-(N-(4-(4-chlorothiophen-2-yl)oxazol-2-

yl)sulfamoyl)acetate (4a) with 4-phenyl-1 H-pyrrol-2-amine (7a) in the presence of two molar equivalents of sodium methoxide in THF under ultrasonication at a frequency $35 \mathrm{KHz}$. The product $\mathrm{N}$-(4-(4-chlorothiophen-2-yl)oxazol-2yl)-2-( $N$-(4-phenyl-1 H-pyrrol-2-yl)sulfamoyl)acetamide (8a) was obtained in low yield (38\%). However when the reaction was repeated with three molar equivalents, the yield of $\mathbf{8 a}$ was raised to $48 \%$. In order to increase the yield, the reaction between $\mathbf{4 a}$ and $7 \mathbf{a}$ was carried out with different molar concentration of sodium methoxide. The reaction took place well in the presence of 5.0 mol\% sodium methoxide leading to the formation of product $8 \mathbf{a}$ in excellent yield (Table 1).

Then the reaction of $\mathbf{4 , 5}$ and $\mathbf{6}$ was carried out with 4-aryl-1/-pyrrol-2-amine (7) in the presence of $5.0 \mathrm{~mol} \%$ sodium methoxide to get $N$-(4-(4-chlorothiophen-2-yl)oxazol-2-yl)-2-( $N$-(4-phenyl-1 H-pyrrol-2-

yl) sulfamoyl)acetamide (8) / N-(4-(4-chlorothiophen-2-yl)thiazol-2-yl)-2-(N-(4-phenyl-1 H-pyrrol-2-

yl)sulfamoyl)acetamide (9) / N-(4-(4-chlorothiophen-2-yl)-1 H-imidazol-2-yl)-2-(N-(4-phenyl-1H-pyrrol-2-

yl)sulfamoyl)acetamide (10) (Scheme-II).

\section{Biological evaluation}

\section{Antibacterial activity}

All the compounds are tested for antibacterial activity at four different concentrations $12.5,25,50$, and 100 $\mu \mathrm{g} /$ well. All the compounds displayed higher activity on Gram-positive bacteria than on Gram-negative bacteria (Table 1). Thiophenylthiazolyl pyrrolylsulfamoyl acetamides (9) showed higher activity than thiophenyloxazolyl pyrrolylsulfamoyl acetamides (8) and thiophenylimidazolyl pyrrolylsulfamoyl acetamides (10). Amongst the latter compounds $\mathbf{1 0}$ exhibited greater activity than $\mathbf{8}$. It was also observed that the presence of electron withdrawing substituents enhanced the activity and the activity increased with increasing electronegativity. The compounds having nitro substituents displayed higher activity than those with chloro substituents. In fact, dinitro substituted compounds $\mathbf{8 f}$, $9 \mathbf{f}$ and $\mathbf{1 0 f}$ displayed higher activity in the respective series. It was also observed that those having chloro and nitro substituents $\mathbf{8 d}, \mathbf{9 d}, \mathbf{1 0 d}$ and $\mathbf{8 e}, \mathbf{9 e}, \mathbf{1 0 e}$ showed slightly higher activity when compared with the 
compounds having two chloro substituents $8 c, 9 c$ and $10 c$. Further it was noticed that there was not much difference in activity amongst the compounds $\mathbf{8 d}, \mathbf{9 d}, \mathbf{1 0 d}$ and $\mathbf{8 e}, \mathbf{9 e}, \mathbf{1 0} \mathbf{e}$ which showed that the presence of more electron withdrawing nitro substituent either at 4 position of thiophene or at 4 position of aromatic ring has similar effect. Amongst all the tested compounds $\mathbf{9 d}, \mathbf{9 e}, \mathbf{9 f}$ and $\mathbf{1 0 f}$ displayed higher activity on $B$. subtilis greater than the standard drug Chloramphenicol at all tested concentrations. Further the compound $9 \mathrm{f}$ displayed equal activity to the standard drug on $S$. aureus at 50 and $100 \mu \mathrm{g} /$ well.

\section{Antifungal activity}

All the compounds effectively inhibited the spore germination of tested fungi (Table 2). It was noticed that compounds having imidazole unit (10) displayed grater activity than those with oxazole (8) and thiazole (9) moieties. Further the activity increased with increasing electronegativity of the substituents. The compounds $\mathbf{9 f}$, $10 \mathrm{~d}, 10 \mathrm{e}$ and $10 \mathrm{f}$ showed higher activity on A. niger greater than the standard drug, Ketoconazole at all tested concentrations.

The compounds which showed greater antibacterial and antifungal activities are further assayed for minimum inhibitory concentration (MIC), minimum bactericidal concentration (MBC) and minimum fungicidal concentration (MFC) and the values are listed in Table 3. MIC is the lowest concentration of an antimicrobial that will inhibit the visible growth of a microorganism. (But it is not sure that the microorganisms are completely killed). The $\mathrm{MBC} / \mathrm{MFC}$ is the lowest concentration of antibiotic required to kill a particular bacterium/fungi. The MBC/MFC involves an additional set of steps performed once the MIC is determined. The antimicrobials are usually regarded as bactericidal/fungicidal if MBC/MFC is not greater than four times MIC. The compounds $\mathbf{9 d}, \mathbf{9 e}, \mathbf{9 f}$ and $\mathbf{1 0 f}$ displayed low MIC against $B$. Subtilis. The MIC of these compounds is equal to standard drug chloramphenicol and MBC is $2 \times$ MIC. Further 9 f showed low MIC on S. aureus and MBC is found to be $2 \times$ MIC. Moreover the compounds $\mathbf{9 f}, \mathbf{1 0 d}, \mathbf{1 0 e}$ and $\mathbf{1 0 f}$ exhibited low MIC against $A$. niger and MFC is 2 x MIC.

\section{Conclusion}

A variety of thiophenylazolyl pyrrolylsulfamoyl acetamides were prepared by the reaction of azolylsulfamoyl acetate with pyrrolylamine in the presence of sodium methoxide in methanol under ultrasonication. The presence of electron withdrawing substituents increased antimicrobial activity. Chloro, nitro and dinitro substituted thiophenylthiazolyl pyrrolylsulfamoyl acetamides (9d, 9e, 9f) and dinitro substituted thiophenylimidazolyl pyrrolylsulfamoyl acetamide (10f) exhibited potential antibacterial activity against $B$. subtilis. These compounds exhibited low MIC against $B$. Subtilis equal to standard drug chloramphenicol and MBC is $2 \times$ MIC. Further $9 f$ showed low MIC on S. aureus and MBC is $2 \times$ MIC. The compounds $9 \mathrm{f}, 10 \mathrm{~d}, 10 \mathrm{e}$ and $10 \mathrm{f}$ displayed prominent antifungal activity against $A$. niger and MFC is $2 \times$ MIC.

\section{Experimental Section}

\section{Apparatus and analysis}

Melting points were determined in open capillaries on a Mel-Temp apparatus and are uncorrected. The purity of the compounds was evaluated by TLC (silica gel $\mathrm{H}, \mathrm{BDH}$, ethyl acetate/hexane, 1:3). The IR spectra were recorded on a Thermo Nicolet IR $200 \mathrm{FT}-\mathrm{IR}$ spectrometer as $\mathrm{KBr}$ pellets and the wave numbers were given in $\mathrm{cm}^{-1}$. The ${ }^{1} \mathrm{H}$ and

${ }^{13} \mathrm{C}$ NMR spectra were recorded in DMSO- $\mathrm{d}_{6}$ on a Bruker spectrometer operating at 400 and $100 \mathrm{MHz}$. The 
chemical shifts are reported in $\delta(\mathrm{ppm})$ using TMS as an internal standard. The high-resolution mass spectra are recorded on micromass Q-TOF micromass spectrometer using electrospray ionization. Ultrasonication was performed in a Bandelinsonorex RK $12 \mathrm{H}$ ultrasonic bath operating at a frequency of $35 \mathrm{KHz}$. The microanalyses were performed on a Perkin-Elmer 240C elemental analyzer. The progress of the reaction was monitored by TLC using silica gel plates (silica gel 60 F254 0.25 mm), and components were visualized by observation under UV light (254 and $365 \mathrm{~nm}$ ). The compounds 4-thiophenyloxazolyl-2-amine (1) and 4-thiophenylthiazolyl-2-amine (2) 4thiophenylimidazolyl-2-amine (3) were prepared as per the literature procedures [Taterao et al. 2008]. The compound 4-(4-chlorophenyl)-1H-pyrrol-2-amine (7) was purchased from Aldrich.

\section{Preparation of dispersed sodium}

Clean sodium metal $(10 \mathrm{~g})$ was weighed under dry ether and introduced into a $500 \mathrm{ml}$ round-bottomed flask containing sodium-dried xylene $\left(100 \mathrm{~cm}^{3}\right)$ fitted with an air condenser carrying a calcium chloride guard tube and placed on a sand bath. The flask was enveloped with a dry cloth and the sand bath was heated cautiously. The ring of condensed vapour of xylene was carefully observed. When the ring of condensed vapor had risen to the neck of the flask the flame was extinguished. The condenser was replaced by the stopper and the flask was wrapped with a pre-dried cloth. The stopper was then held firmly and shaken vigorously for 2-3 min until the molten sodium was converted into a fine dispersion. Immediately the stopper was removed and the flask was placed on the cork ring. The sodium was obtained in the form of small spheres depending upon the time and rapidity of shaking. Then the contents were cooled to room temperature, xylene was decanted and the sodium was washed with sodium-dried ether. The dispersed sodium as small spheres was preserved in absolute ether.

General procedure for the synthesis of methyl 4-thiophenylazolylsulfamoyl acetate (4/5/6)

A mixture of methyl 2-chlorosulfonylacetate $(0.001 \mathrm{~mol})$, dispersed sodium $(0.0414 \mathrm{~g}, 1.8 \mathrm{mg}$ atom) and tetrahydrofuran $\left(3 \mathrm{ml}\right.$ ) was sonicated for $8 \mathrm{~min}$ in a sonic bath at a frequency of $35 \mathrm{KHz}$ at $25^{0} \mathrm{C}$. To this azolyl-2amine (1/2/3) was added and continued sonication for 12-16 min. After completion of the reaction (monitored by TLC), the organic matter was filtered, washed with water, extracted with ether and dried. Removal of the solvent under reduced pressure gave a solid which was recrystallized from ethanol.

Methyl 2-(N-(4-(4-chlorothiophen-2-yl)oxazol-2-yl)sulfamoyl)acetate (4a). Yield 75\%; m.p.: 122-124이 $\mathrm{IR}(\mathrm{KBr})$ : 1129, $1327\left(\mathrm{SO}_{2}\right), 1579(\mathrm{C}=\mathrm{N}), 1632(\mathrm{C}=\mathrm{C}), 1680(\mathrm{C}=0), 3356(\mathrm{NH})\left(\mathrm{cm}^{-1}\right) ;{ }^{1} \mathrm{H}$ NMR $\left(400 \mathrm{MHz}, \mathrm{DMSO}-d_{6}\right): \delta 3.68$ $\left(\mathrm{s}, 3 \mathrm{H}, 0-\mathrm{CH}_{3}\right), 4.36\left(\mathrm{~s}, 2 \mathrm{H}, \mathrm{CH}_{2}\right), 7.07\left(\mathrm{~s}, 1 \mathrm{H}, \mathrm{C}_{5^{-}}-\mathrm{H}\right), 7.35\left(\mathrm{~s}, 1 \mathrm{H}, \mathrm{C}_{3^{-}}-\mathrm{H}\right), 7.58\left(\mathrm{~s}, 1 \mathrm{H}, \mathrm{C}_{5}-\mathrm{H}\right), 10.46\left(\mathrm{bs}, 1 \mathrm{H}, \mathrm{SO}_{2} \mathrm{NH}\right) \mathrm{ppm}$ ${ }^{13} \mathrm{C}$ NMR $\left(100 \mathrm{MHz}\right.$, DMSO- $\left.d_{6}\right): \delta 49.1\left(\mathrm{O}-\mathrm{CH}_{3}\right), 70.4\left(\mathrm{CH}_{2}\right), 121.9,123.7,125.4,127.2,141.5,145.1,146.6$ (Aromatic carbons C-2, C-4, C-5, C-2', C-3', C-4' \& C-5'), 163.0 (C= 0) ppm; HRMS (m/z): 359.7513 [M + Na]; Anal. Calcd. for $\mathrm{C}_{10} \mathrm{H}_{9} \mathrm{CIN}_{2} \mathrm{O}_{5} \mathrm{~S}_{2}$ : C, 35.67; $\mathrm{H}, 2.69 ; \mathrm{N}, 8.32$; Found: C, 35.74; $\mathrm{H}, 2.63 ; \mathrm{N}, 8.41 \%$.

Methyl 2-(N-(4-(4-nitrothiophen-2-yl)oxazol-2-yl)sulfamoyl)acetate (4b). Yield 72\%; m.p.: 145-147 C; IR (KBr): 1135, $1321\left(\mathrm{SO}_{2}\right), 1587(\mathrm{C}=\mathrm{N}), 1650(\mathrm{C}=\mathrm{C}), 1676(\mathrm{C}=0), 3367(\mathrm{NH})\left(\mathrm{cm}^{-1}\right) ;{ }^{1} \mathrm{H} \mathrm{NMR}\left(400 \mathrm{MHz}, \mathrm{DMSO}-d_{6}\right): \delta 3.64(\mathrm{~s}$, $\left.3 \mathrm{H}, 0-\mathrm{CH}_{3}\right), 4.34\left(\mathrm{~s}, 2 \mathrm{H}, \mathrm{CH}_{2}\right), 7.61\left(\mathrm{~s}, 1 \mathrm{H}, \mathrm{C}_{5}-\mathrm{H}\right), 8.39\left(\mathrm{~s}, 1 \mathrm{H}, \mathrm{C}_{5^{\prime}}-\mathrm{H}\right), 8.66\left(\mathrm{~s}, 1 \mathrm{H}, \mathrm{C}_{3^{\prime}}-\mathrm{H}\right), 10.48\left(\mathrm{bs}, 1 \mathrm{H}, \mathrm{SO}_{2} \mathrm{NH}\right) \mathrm{ppm}$;

${ }^{13} \mathrm{C}$ NMR $(100 \mathrm{MHz}$, DMSO-d 6$): \delta 51.9\left(0-\mathrm{CH}_{3}\right), 65.6\left(\mathrm{CH}_{2}\right), 117.3,127.1,132.6,138.0,146.5,149.4,153.2$ (Aromatic carbons C-2, C-4, C-5, C-2', C-3', C-4' \& C-5'), 162.8 (C= 0) ppm; HRMS (m/z): 370.3051 [M + Na]; Anal. Calcd. for $\mathrm{C}_{10} \mathrm{H}_{9} \mathrm{~N}_{3} \mathrm{O}_{7} \mathrm{~S}_{2}$ : C, 34.58; $\mathrm{H}, 2.61 ; \mathrm{N}, 12.10$; Found: $\mathrm{C}, 34.50 ; \mathrm{H}, 2.66 ; \mathrm{N}, 12.26 \%$. 
Methyl 2-(N-(4-(4-chlorothiophen-2-yl)thiazol-2-yl)sulfamoyl)acetate (5a). Yield 76\%; m.p.: 138-140 ${ }^{\circ} \mathrm{C}$; IR (KBr): 1143, $1335\left(\mathrm{SO}_{2}\right), 1583(\mathrm{C}=\mathrm{N}), 1643(\mathrm{C}=\mathrm{C}), 1688(\mathrm{C}=0), 3365(\mathrm{NH})\left(\mathrm{cm}^{-1}\right) ;{ }^{1} \mathrm{H}$ NMR $\left(400 \mathrm{MHz}, \mathrm{DMSO}_{6}\right): \delta 3.70$ $\left(\mathrm{s}, 3 \mathrm{H}, 0-\mathrm{CH}_{3}\right), 4.32\left(\mathrm{~s}, 2 \mathrm{H}, \mathrm{CH}_{2}\right), 7.02\left(\mathrm{~s}, 1 \mathrm{H}, \mathrm{C}_{5}-\mathrm{H}\right), 7.10\left(\mathrm{~s}, 1 \mathrm{H}, \mathrm{C}_{5^{\prime}} \mathrm{H}\right), 7.41\left(\mathrm{~s}, 1 \mathrm{H}, \mathrm{C}_{3^{\prime}}-\mathrm{H}\right), 10.44\left(\mathrm{bs}, 1 \mathrm{H}, \mathrm{sO}_{2} \mathrm{NH}\right) \mathrm{ppm}$; ${ }^{13} \mathrm{C}$ NMR $\left(100 \mathrm{MHz}\right.$, DMSO- $\left.d_{6}\right): \delta 49.7\left(\mathrm{O}-\mathrm{CH}_{3}\right), 63.2\left(\mathrm{CH}_{2}\right), 105.2(\mathrm{C}-5), 124.3,127.0,131.5,140.3,145.1$ (Aromatic carbons C-4, C-2', C-3', C-4' \& C-5'), 162.6 (C= 0), 165.4 (C-2) ppm; HRMS (m/z): 375.8122 [M + Na]; Anal. Calcd. for $\mathrm{C}_{10} \mathrm{H}_{9} \mathrm{ClN}_{2} \mathrm{O}_{4} \mathrm{~S}_{3}:$ C, 34.04; $\mathrm{H}, 2.57 ; \mathrm{N}, 7.94$; Found: C, 33.98; $\mathrm{H}, 2.62 ; \mathrm{N}, 7.99 \%$.

Methyl 2-(N-(4-(4-nitrothiophen-2-yl)thiazol-2-yl)sulfamoyl)acetate (5b). Yield 74\%; m.p.: 163-165 $\mathrm{C}$; IR (KBr): 1124, $1341\left(\mathrm{SO}_{2}\right), 1578(\mathrm{C}=\mathrm{N}), 1652(\mathrm{C}=\mathrm{C}), 1674(\mathrm{C}=0), 3371(\mathrm{NH})\left(\mathrm{cm}^{-1}\right) ;{ }^{1} \mathrm{H} \mathrm{NMR}\left(400 \mathrm{MHz}, \mathrm{DMSO}-d_{6}\right): \delta 3.74(\mathrm{~s}$, $\left.3 \mathrm{H}, 0-\mathrm{CH}_{3}\right), 4.36\left(\mathrm{~s}, 2 \mathrm{H}, \mathrm{CH}_{2}\right), 7.05\left(\mathrm{~s}, 1 \mathrm{H}, \mathrm{C}_{5}-\mathrm{H}\right), 8.35\left(\mathrm{~s}, 1 \mathrm{H}, \mathrm{C}_{5^{\prime}}-\mathrm{H}\right), 8.69\left(\mathrm{~s}, 1 \mathrm{H}, \mathrm{C}_{3^{\prime}}-\mathrm{H}\right), 10.42\left(\mathrm{bs}, 1 \mathrm{H}, \mathrm{SO}_{2} \mathrm{NH}\right) \mathrm{ppm}$; ${ }^{13} \mathrm{C}$ NMR $\left(100 \mathrm{MHz}, \mathrm{DMSO}-d_{6}\right): \delta 50.2\left(\mathrm{O}-\mathrm{CH}_{3}\right), 64.5\left(\mathrm{CH}_{2}\right), 106.7$ (C-5), $117.2(\mathrm{C}-3), 128.7,144.5,146.4,152.3$ (Aromatic carbons C-4, C-2', C-4' \& C-5'), 162.1 (C= 0), 169.6 (C-2) ppm; HRMS (m/z): 386.3673 [M + Na]; Anal. Calcd. for $\mathrm{C}_{10} \mathrm{H}_{9} \mathrm{~N}_{3} \mathrm{O}_{6} \mathrm{~S}_{3}$ : C, 33.05; $\mathrm{H}, 2.50 ; \mathrm{N}, 11.56$; Found: $\mathrm{C}, 33.12 ; \mathrm{H}, 2.54 ; \mathrm{N}, 11.65 \%$.

Methyl 2-(N-(4-(4-chlorothiophen-2-yl)-1H-imidazol-2-yl)sulfamoyl)acetate (6a). Yield 77\%; m.p.: 149-151 ${ }^{\circ} \mathrm{C}$; IR $(\mathrm{KBr}): 1148,1330\left(\mathrm{SO}_{2}\right), 1585(\mathrm{C}=\mathrm{N}), 1645(\mathrm{C}=\mathrm{C}), 1687(\mathrm{C}=0), 3377(\mathrm{NH})\left(\mathrm{cm}^{-1}\right) ;{ }^{1} \mathrm{H}$ NMR $\left(400 \mathrm{MHz}, \mathrm{DMSO}-d_{6}\right)$ : $\delta 3.69\left(\mathrm{~s}, 3 \mathrm{H}, 0-\mathrm{CH}_{3}\right), 4.31\left(\mathrm{~s}, 2 \mathrm{H}, \mathrm{CH}_{2}\right), 7.06\left(\mathrm{~s}, 1 \mathrm{H}, \mathrm{C}_{5}-\mathrm{H}\right), 7.37\left(\mathrm{~s}, 1 \mathrm{H}, \mathrm{C}_{3^{-}}-\mathrm{H}\right), 7.40\left(\mathrm{~s}, 1 \mathrm{H}, \mathrm{C}_{5}-\mathrm{H}\right), 10.38(\mathrm{bs}, 1 \mathrm{H}$, $\left.\mathrm{SO}_{2} \mathrm{NH}\right), 12.88$ (bs, $1 \mathrm{H}$, Imidazole NH) ppm; ${ }^{13} \mathrm{C}$ NMR (100 MHz, DMSO-d $): \delta 51.8\left(0-\mathrm{CH}_{3}\right), 61.0\left(\mathrm{CH}_{2}\right), 121.3$, 123.1, 125.1, 128.6, 139.4, 143.2, 154.5 (Aromatic carbons C-2, C-4, C-5, C-2', C-3', C-4' \& C-5'), 163.7 (C= O) ppm; $\operatorname{HRMS}(\mathrm{m} / \mathrm{z}): 358.7672$ [M + Na]; Anal. Calcd. for $\mathrm{C}_{10} \mathrm{H}_{10} \mathrm{ClN}_{3} \mathrm{O}_{4} \mathrm{~S}_{2}: \mathrm{C}, 35.77 ; \mathrm{H}, 3.00 ; \mathrm{N}, 12.51$; Found: $\mathrm{C}, 35.84 ; \mathrm{H}$, $3.04 ; \mathrm{N}, 12.44 \%$.

Methyl 2-(N-(4-(4-nitrothiophen-2-yl)-1H-imidazol-2-yl)sulfamoyl)acetate (6b). Yield 75\%; m.p.: 178-180 ${ }^{\circ} \mathrm{C}$; IR (KBr): 1136, $1322\left(\mathrm{SO}_{2}\right), 1588(\mathrm{C}=\mathrm{N}), 1633(\mathrm{C}=\mathrm{C}), 1675(\mathrm{C}=0), 3380(\mathrm{NH})\left(\mathrm{cm}^{-1}\right) ;{ }^{1} \mathrm{H} \mathrm{NMR}\left(400 \mathrm{MHz}, \mathrm{DMSO}_{-} d_{6}\right): \delta 3.73$ $\left(\mathrm{s}, 3 \mathrm{H}, 0-\mathrm{CH}_{3}\right), 4.38\left(\mathrm{~s}, 2 \mathrm{H}, \mathrm{CH}_{2}\right), 7.42\left(\mathrm{~s}, 1 \mathrm{H}, \mathrm{C}_{5}-\mathrm{H}\right), 8.38\left(\mathrm{~s}, 1 \mathrm{H}, \mathrm{C}_{5^{-}}-\mathrm{H}\right), 8.65\left(\mathrm{~s}, 1 \mathrm{H}, \mathrm{C}_{3^{\prime}}-\mathrm{H}\right), 10.40\left(\mathrm{bs}, 1 \mathrm{H}, \mathrm{SO}_{2} \mathrm{NH}\right)$, 12.90 (bs, $1 \mathrm{H}$, Imidazole NH) ppm; ${ }^{13} \mathrm{C}$ NMR (100 MHz, DMSO-d 6$): \delta 52.3\left(0-\mathrm{CH}_{3}\right), 62.1\left(\mathrm{CH}_{2}\right), 122.9,124.8,133.4$, 139.0, 146.8, 152.9, 154.1 (Aromatic carbons C-2, C-4, C-5, C-2', C-3', C-4' \& C-5'), 165.4 (C = 0) ppm; HRMS (m/z): 369.3224 [M + Na]; Anal. Calcd. for $\mathrm{C}_{10} \mathrm{H}_{10} \mathrm{~N}_{4} \mathrm{O}_{6} \mathrm{~S}_{2}$ : C, 34.68; $\mathrm{H}, 2.91 ; \mathrm{N}, 16.18$; Found: C, 34.62; $\mathrm{H}, 2.62 ; \mathrm{N}, 16.15 \%$

General procedure for the synthesis of N-(4-(4-chlorothiophen-2-yl)oxazol / thiazol / imidazol-2-yl)-2-(N-(4-phenyl$1 \mathrm{H}-$ pyrrol-2-yl)sulfamoyl) acetamide (8/9/10)

A mixture of sodium methoxide (0.0011 mol), 4-phenyl-1 H-pyrrol-2-amine (7) and methanol (5 ml) was sonicated at a frequency of $35 \mathrm{KHz}$ at room temperature for $5 \mathrm{~min}$. To this thiophenyl azolyl sulfamoyl acetate (4/5/6) (0.001 $\mathrm{mol}$ ) was added and continued sonication for 22-30 min. The solvent was evaporated under reduced pressure. The gummy substance was recrystallized from ethanol.

N-(4-(4-Chlorothiophen-2-yl)oxazol-2-yl)-2-(N-(4-phenyl-1H-pyrrol-2-yl)sulfamoyl)acetamide (8a) yield 64\% ; m.p.: 149-151 ${ }^{\circ} \mathrm{C}$; IR (KBr): 3375 (CONH), 1712 (C = O), 1678 (C = C), $1579(\mathrm{C}=\mathrm{N}), 1326-1132\left(\mathrm{SO}_{2}\right)\left(\mathrm{cm}^{-1}\right) ;{ }^{1} \mathrm{H} \mathrm{NMR}$ $\left(400 \mathrm{MHz}, \mathrm{DMSO}-d_{6}\right): \delta 4.28\left(\mathrm{~s}, 2 \mathrm{H}, \mathrm{CH}_{2}\right), 6.60\left(\mathrm{~s}, 1 \mathrm{H}, \mathrm{C}_{3^{\prime}}-\mathrm{H}\right), 7.05\left(\mathrm{~s}, 1 \mathrm{H}, \mathrm{C}_{5^{1}}-\mathrm{H}\right), 7.14\left(\mathrm{~s}, 1 \mathrm{H}, \mathrm{C}_{5^{-}} \mathrm{H}\right), 7.40-7.66(\mathrm{~m}, 7 \mathrm{H}$, Ar-H, $\left.\mathrm{C}_{3^{2}}-\mathrm{H} \& \mathrm{C}_{5}-\mathrm{H}\right), 9.92$ (bs, $\left.1 \mathrm{H}, \mathrm{CONH}_{2}\right), 10.32$ (bs, $\left.1 \mathrm{H}, \mathrm{NH}\right), 11.45$ (bs, $1 \mathrm{H}$, Pyrrole NH) ppm; ${ }^{13} \mathrm{C}$ NMR $(100 \mathrm{MHz}$, DMSO- $\left.d_{6}\right)$ : $\delta 64.2\left(\mathrm{H}_{2} \mathrm{C}-\mathrm{CO}\right), 104.1$ (C-3"), 116.3 (C-5"), 118.8 (C-5'), $144.0\left(\mathrm{C}-2^{\prime}\right), 151.4(\mathrm{C}-2), 169.8$ (C = 0), 121.3, 122.1, 123.5, 125.9, 126.7, 127.8, 133.3, 136.1, 138.5, 139.7 (Aromatic carbons \& C-4, C-5, C-3', C-4', C-2", C-4") ppm. 
HRMS $(\mathrm{m} / \mathrm{z}): 485.9126[\mathrm{M}+\mathrm{Na}]^{+}$; Anal. Calcd. for $\mathrm{C}_{19} \mathrm{H}_{15} \mathrm{CIN}_{4} \mathrm{O}_{4} \mathrm{~S}_{2}: \mathrm{C}, 49.30 ; \mathrm{H}, 3.27 ; \mathrm{N}, 12.10 ;$ Found: $\mathrm{C}, 49.39 ; \mathrm{H}$, $3.35 ; \mathrm{N}, 12.18 \%$.

N-(4-(4-Nitrothiophen-2-yl)oxazol-2-yl)-2-(N-(4-phenyl-1H-pyrrol-2-yl)sulfamoyl)acetamide (8b) yield 68\% ; m.p.: 153155다 IR (KBr): $3381(\mathrm{CONH}), 1688$ (C = O), 1679 (C= C), $1577(\mathrm{C}=\mathrm{N}), 1332-1140\left(\mathrm{SO}_{2}\right)\left(\mathrm{cm}^{-1}\right) ;{ }^{1} \mathrm{H}$ NMR $(400$

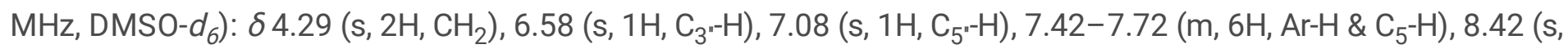
$\left.1 \mathrm{H}, \mathrm{C}_{5}-\mathrm{H}\right), 8.79\left(\mathrm{~s}, 1 \mathrm{H}, \mathrm{C}_{3}-\mathrm{H}\right), 9.95$ (bs, $\left.1 \mathrm{H}, \mathrm{CONH}_{2}\right), 10.49$ (bs, $\left.1 \mathrm{H}, \mathrm{NH}\right), 11.66$ (bs, $1 \mathrm{H}$, Pyrrole NH) ppm; ${ }^{13} \mathrm{C} \mathrm{NMR}$ $\left(100 \mathrm{MHz}, \mathrm{DMSO}-d_{6}\right): \delta 65.1\left(\mathrm{H}_{2} \mathrm{C}-\mathrm{CO}\right), 106.6$ (C-3"), 116.7 (C-5"), 118.5 (C-3'), 124.1 (C-4), 144.3 (C-2'), 149.1 (C-4'), 152.6 (C-2), 170.4 (C = 0), 125.8, 128.5, 130.7, 132.4, 134.9, 135.6, 136.8, 137.6 (Aromatic carbons \& C-5, C-5', C-2", C-4") ppm. HRMS (m/z): 496.4676 [M + Na] ${ }^{+}$; Anal. Calcd. for $\mathrm{C}_{19} \mathrm{H}_{15} \mathrm{~N}_{5} \mathrm{O}_{6} \mathrm{~S}_{2}: \mathrm{C}, 48.20 ; \mathrm{H}, 3.19 ; \mathrm{N}, 14.79$; Found: C, 48.27; H, 3.14; N, 14.68\%.

2-(N-(4-(4-Chlorophenyl)-1H-pyrrol-2-yl)sulfamoyl)-N-(4-(4-chlorothiophen-2-yl)oxazol-2-yl) acetamide (8c) yield 67\% ; m.p.: 159-161 ${ }^{\circ} \mathrm{C}$; IR (KBr): $3377(\mathrm{CONH}), 1722(\mathrm{C}=\mathrm{O}), 1680(\mathrm{C}=\mathrm{C}), 1582(\mathrm{C}=\mathrm{N}), 1335-1132\left(\mathrm{SO}_{2}\right)\left(\mathrm{cm}^{-1}\right) ;{ }^{1} \mathrm{H}$ $\operatorname{NMR}\left(400 \mathrm{MHz}, \mathrm{DMSO}-d_{6}\right): \delta 4.27\left(\mathrm{~s}, 2 \mathrm{H}, \mathrm{CH}_{2}\right), 6.56\left(\mathrm{~s}, 1 \mathrm{H}, \mathrm{C}_{3^{-}} \mathrm{H}\right), 7.04\left(\mathrm{~s}, 1 \mathrm{H}, \mathrm{C}_{5^{-}} \mathrm{H}\right), 7.15\left(\mathrm{~s}, 1 \mathrm{H}, \mathrm{C}_{5^{-}} \mathrm{H}\right), 7.58-7.84$ (m, 6H, Ar- $\left.\mathrm{H} \mathrm{C}_{5}-\mathrm{H} \& \mathrm{C}_{3^{-}}-\mathrm{H}\right), 10.26$ (bs, $\left.1 \mathrm{H}, \mathrm{CONH}_{2}\right), 10.41$ (bs, $\left.1 \mathrm{H}, \mathrm{NH}\right), 11.81$ (bs, $1 \mathrm{H}$, Pyrrole NH) ppm; ${ }^{13} \mathrm{C} \mathrm{NMR}(100$ MHz, DMSO- $\left.d_{6}\right): \delta 65.2\left(\mathrm{H}_{2} \mathrm{C}-\mathrm{CO}\right), 106.3$ (C-3"), 117.9 (C-5"), 119.2 (C-3'), 121.2 (C-5'), 140.7 (C-5), 145.6 (C-2'), 154.3 (C-2), 170.3 (C = 0), 125.1, 126.3, 127.2, 130.5, 132.2, 135.4, 136.5, 137.3 (Aromatic carbons \& C-4, C-4', C-2", C-4") ppm; HRMS (m/z): $520.3850\left[\mathrm{M}+\mathrm{Na}^{+}\right.$; Anal. Calcd. for $\mathrm{C}_{19} \mathrm{H}_{14} \mathrm{Cl}_{2} \mathrm{~N}_{4} \mathrm{O}_{4} \mathrm{~S}_{2}$ : C, 45.88; $\mathrm{H}, 2.84 ; \mathrm{N}, 11.26$; Found: C, 45.80; H, 2.80; N, $11.18 \%$.

2-(N-(4-(4-Chlorophenyl)-1H-pyrrol-2-yl)sulfamoyl)-N-(4-(4-nitrothiophen-2-yl)oxazol-2-yl) acetamide (8d) yield 70\%; m.p.: $165-167^{\circ} \mathrm{C}$; IR (KBr): $3372(\mathrm{CONH}), 1702(\mathrm{C}=\mathrm{O}), 1684(\mathrm{C}=\mathrm{C}), 1568(\mathrm{C}=\mathrm{N}), 1335-1128\left(\mathrm{SO}_{2}\right)\left(\mathrm{cm}^{-1}\right) ;{ }^{1} \mathrm{H}$

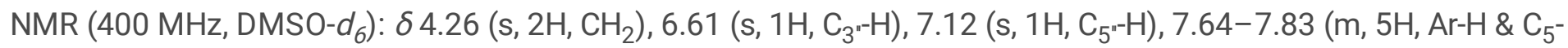
$\mathrm{H}), 8.44\left(\mathrm{~s}, 1 \mathrm{H}, \mathrm{C}_{5^{-}} \mathrm{H}\right), 8.75\left(\mathrm{~s}, 1 \mathrm{H}, \mathrm{C}_{3^{-}}-\mathrm{H}\right), 10.20$ (bs, $\left.1 \mathrm{H}, \mathrm{CONH}_{2}\right), 10.48$ (bs, $\left.1 \mathrm{H}, \mathrm{NH}\right), 11.93$ (bs, 1H, Pyrrole NH) ppm; ${ }^{13} \mathrm{C}$ NMR (100 MHz, DMSO-d $)$ : $\delta 64.5$ (H $\left.\mathrm{H}_{2} \mathrm{C}-\mathrm{CO}\right), 107.2$ (C-3"), 114.5 (C-3'), 117.3 (C-5"), 142.8 (C-2'), 154.1 (C-2), $172.1(\mathrm{C}=0), 149.8\left(\mathrm{C}-4{ }^{\prime}\right), 123.1,125.2,127.4,128.8,129.5,132.6,134.1,137.9,139.2$ (Aromatic carbons, C-4, C-5, C-5', C-2" \& C-4") ppm. HRMS (m/z): $530.9095[\mathrm{M}+\mathrm{Na}]^{+}$; Anal. Calcd. for $\mathrm{C}_{19} \mathrm{H}_{14} \mathrm{ClN}_{5} \mathrm{O}_{6} \mathrm{~S}_{2}: \mathrm{C}, 44.93 ; \mathrm{H}, 2.78 ; \mathrm{N}$, 13.79; Found: C, 45.00; H, 2.74; N, $13.85 \%$.

N-(4-(4-Chlorothiophen-2-yl)oxazol-2-yl)-2-(N-(4-(4-nitrophenyl)-1H-pyrrol-2-yl)sulfamoyl) acetamide (8e) yield 66\%; m.p.: 160-162 ${ }^{\circ} \mathrm{C}$; IR (KBr): $3380(\mathrm{CONH}), 1721(\mathrm{C}=\mathrm{O}), 1676(\mathrm{C}=\mathrm{C}), 1575(\mathrm{C}=\mathrm{N}), 1339-1141\left(\mathrm{SO}_{2}\right)\left(\mathrm{cm}^{-1}\right) ;{ }^{1} \mathrm{H}$ $\operatorname{NMR}\left(400 \mathrm{MHz}, \mathrm{DMSO}-d_{6}\right): \delta 4.20\left(\mathrm{~s}, 2 \mathrm{H}, \mathrm{CH}_{2}\right), 6.74\left(\mathrm{~s}, 1 \mathrm{H}, \mathrm{C}_{3^{-1}} \mathrm{H}\right), 7.13\left(\mathrm{~s}, 1 \mathrm{H}, \mathrm{C}_{5^{n}}-\mathrm{H}\right), 7.19\left(\mathrm{~s}, 1 \mathrm{H}, \mathrm{C}_{5^{\prime}}-\mathrm{H}\right), 7.59-7.75$ $\left(\mathrm{m}, 6 \mathrm{H}, \mathrm{Ar}-\mathrm{H}, \mathrm{C}_{3^{\prime}}-\mathrm{H} \& \mathrm{C}_{5}-\mathrm{H}\right), 10.15$ (bs, $\left.1 \mathrm{H}, \mathrm{CONH}_{2}\right), 10.65$ (bs, $\left.1 \mathrm{H}, \mathrm{NH}\right), 12.04$ (bs, $\left.1 \mathrm{H}, \mathrm{Pyrrole} \mathrm{NH}\right) \mathrm{ppm} ;{ }^{13} \mathrm{C} \mathrm{NMR}$ (100 MHz, DMSO- $\left.d_{6}\right): \delta 65.8\left(\mathrm{H}_{2} \mathrm{C}-\mathrm{CO}\right), 107.4$ (C-3"), $118.3(\mathrm{C}-5 "), 144.3$ (C-2'), 152.5 (C-2), $172.5(\mathrm{C}=0), 124.2$, $126.8,128.5,129.4,130.5,132.3,134.1,135.8,137.6,138.5,139.3$ (Aromatic carbons, C-4, C-5, C-3', C-4', C-5', C-2" \& C-4") ppm. HRMS (m/z): $530.9103[\mathrm{M}+\mathrm{Na}]^{+}$; Anal. Calcd. for $\mathrm{C}_{19} \mathrm{H}_{14} \mathrm{ClN}_{5} \mathrm{O}_{6} \mathrm{~S}_{2}: \mathrm{C}, 44.93 ; \mathrm{H}, 2.78 ; \mathrm{N}, 13.79$; Found: C, $44.85 ; \mathrm{H}, 2.83 ; \mathrm{N}, 13.87 \%$.

2-(N-(4-(4-Nitrophenyl)-7H-pyrrol-2-yl)sulfamoyl)-N-(4-(4-nitrothiophen-2-yl)oxazol-2-yl) acetamide (8f) yield 68\% ; m.p.: $174-176^{\circ} \mathrm{C}$; IR (KBr): $3385(\mathrm{CONH}), 1736(\mathrm{C}=\mathrm{O}), 1687(\mathrm{C}=\mathrm{C}), 1584(\mathrm{C}=\mathrm{N}), 1343-1148\left(\mathrm{SO}_{2}\right)\left(\mathrm{cm}^{-1}\right) ;{ }^{1} \mathrm{H}$

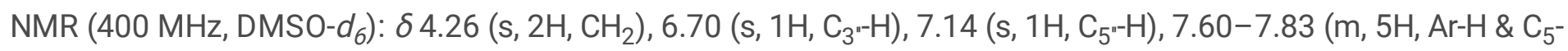


$\mathrm{H}), 8.32\left(\mathrm{~s}, 1 \mathrm{H}, \mathrm{C}_{5^{-}}-\mathrm{H}\right), 8.65\left(\mathrm{~s}, 1 \mathrm{H}, \mathrm{C}_{3^{-}}-\mathrm{H}\right), 10.61$ (bs, 1H, CONH$\left.{ }_{2}\right), 10.72$ (bs,1H, NH), 12.09 (bs, 1H, Pyrrole NH) ppm; ${ }^{13} \mathrm{C}$ NMR (100 MHz, DMSO-d $)$ ): $\delta 66.4$ (H $\left.\mathrm{H}_{2} \mathrm{C}-\mathrm{CO}\right), 107.8$ (C-3"), 117.3 (C-5"), 118.6 (C-3'), $128.4(\mathrm{C}-4), 144.3$ (C-2'), 150.6 (C-4'), 153.9 (C-2), 172.6 (C = 0), 127.7, 128.3, 129.2, 130.4, 133.7, 135.9, 139.8, 140.6 (C-5, C-5', C-2", C-4" \& Aromatic carbons) ppm. HRMS (m/z): $541.4654[\mathrm{M}+\mathrm{Na}]^{+}$; Anal. Calcd. for $\mathrm{C}_{19} \mathrm{H}_{14} \mathrm{~N}_{6} \mathrm{O}_{8} \mathrm{~S}_{2}: \mathrm{C}, 44.02 ; \mathrm{H}, 2.72 ; \mathrm{N}$, 16.21; Found: C, 44.10; H, 2.76; N, $16.34 \%$.

N-(4-(4-Chlorothiophen-2-yl)thiazol-2-yl)-2-(N-(4-phenyl-1H-pyrrol-2-yl)sulfamoyl)acetamide (9a) yield 72\% ; m.p.: 135-137º ; IR (KBr): $3373(\mathrm{CONH}), 1689$ (C = O), 1670 (C = C), $1568(\mathrm{C}=\mathrm{N}), 1335-1130\left(\mathrm{SO}_{2}\right)\left(\mathrm{cm}^{-1}\right) ;{ }^{1} \mathrm{H} \mathrm{NMR}$ (400 MHz, DMSO-d $\left.d_{6}\right): \delta 4.31\left(\mathrm{~s}, 2 \mathrm{H}, \mathrm{CH}_{2}\right), 6.61\left(\mathrm{~s}, 1 \mathrm{H}, \mathrm{C}_{3^{-1}} \mathrm{H}\right), 7.07\left(\mathrm{~s}, 1 \mathrm{H}, \mathrm{C}_{5^{-1}}-\mathrm{H}\right), 7.20-7.68\left(\mathrm{~m}, 7 \mathrm{H}, \mathrm{Ar}-\mathrm{H}, \mathrm{C}_{3^{1}}-\mathrm{H} \& \mathrm{C}_{5^{-}}\right.$ $\mathrm{H}), 7.86$ (s, 1H, $\left.\mathrm{C}_{5}-\mathrm{H}\right), 10.14$ (bs, $\left.1 \mathrm{H}, \mathrm{CONH}\right), 10.46$ (bs, $\left.1 \mathrm{H}, \mathrm{SO}_{2}, \mathrm{NH}\right), 11.52$ (bs, $1 \mathrm{H}$, Pyrrole NH) ppm; ${ }^{13} \mathrm{C} \mathrm{NMR}(100$ MHz, DMSO-d $)_{6}$ : $\delta 64.7$ ( $\left.\mathrm{H}_{2} \mathrm{C}-\mathrm{CO}\right), 104.2$ (C-3"), 105.9 (C-5), 116.5 (C-5"), 119.8 (C-5'), 145.5 (C-2'), 146.3 (C-4), 164.7 (C-2), 170.4 (C = 0), 124.3, 125.2, 127.3, 128.9, 130.5, 133.6, 136.4, 139.2 (Aromatic carbons, C-3', C-4', C-2" \& C-4") ppm. HRMS (m/z): $501.9743[\mathrm{M}+\mathrm{Na}]^{+}$; Anal. Calcd. for $\mathrm{C}_{19} \mathrm{H}_{15} \mathrm{ClN}_{4} \mathrm{O}_{3} \mathrm{~S}_{3}: \mathrm{C}, 47.64 ; \mathrm{H}, 3.16 ; \mathrm{N}, 11.70 ;$ Found: $\mathrm{C}$, $47.70 ; H, 3.20 ; N, 11.78 \%$.

N-(4-(4-Nitrothiophen-2-yl)thiazol-2-yl)-2-(N-(4-phenyl-1H-pyrrol-2-yl)sulfamoyl)acetamide (9b) yield 65\% ; m.p.: 148150 C; IR (KBr): 3379 (CONH), 1718 (C= O), 1671 (C= C), $1573(\mathrm{C}=\mathrm{N}), 1323-1134\left(\mathrm{SO}_{2}\right)\left(\mathrm{cm}^{-1}\right) ;{ }^{1} \mathrm{H} \mathrm{NMR}(400$ $\left.\mathrm{MHz}, \mathrm{DMSO}-d_{6}\right): \delta 4.30\left(\mathrm{~s}, 2 \mathrm{H}, \mathrm{CH}_{2}\right), 6.63\left(\mathrm{~s}, 1 \mathrm{H}, \mathrm{C}_{3^{n}}-\mathrm{H}\right), 7.12\left(\mathrm{~s}, 1 \mathrm{H}, \mathrm{C}_{5}-\mathrm{H}\right), 7.47-7.73(\mathrm{~m}, 5 \mathrm{H}, \mathrm{Ar}-\mathrm{H}), 7.80\left(\mathrm{~s}, 1 \mathrm{H}, \mathrm{C}_{5^{-}}\right.$ $\mathrm{H}), 8.46\left(\mathrm{~s}, 1 \mathrm{H}, \mathrm{C}_{5^{-}} \mathrm{H}\right), 8.84\left(\mathrm{~s}, 1 \mathrm{H}, \mathrm{C}_{3^{-}} \mathrm{H}\right), 10.28\left(\mathrm{bs}, 1 \mathrm{H}, \mathrm{CONH}_{2}\right), 10.52$ (bs, $\left.1 \mathrm{H}, \mathrm{NH}\right), 11.59$ (bs, 1H, Pyrrole NH) ppm; ${ }^{13} \mathrm{C}$ NMR (100 MHz, DMSO- $\left.d_{6}\right): \delta 66.2$ (H $\left.\mathrm{H}_{2} \mathrm{C}-\mathrm{CO}\right), 105.8$ (C-3"), 106.5 (C-5), $119.2(\mathrm{C}-3$ '), 117.6 (C-5"), 144.3 (C-2'), 147.5 (C-4), 149.1 (C-4'), 163.8 (C-2), 171.3 (C = 0), 126.5, 128.2, 129.1, 131.4, 135.2, 136.6, 138.1 (Aromatic carbons, C-2", C-4" \& C-5') ppm. HRMS (m/z): $512.5285[\mathrm{M}+\mathrm{Na}]^{+}$; Anal. Calcd. for $\mathrm{C}_{19} \mathrm{H}_{15} \mathrm{~N}_{5} \mathrm{O}_{5} \mathrm{~S}_{3}: \mathrm{C}, 46.62 ; \mathrm{H}, 3.09$; N, 14.31; Found: C, 46.53; H, 3.05; N, $14.39 \%$.

2-(N-(4-(4-Chlorophenyl)-1H-pyrrol-2-yl)sulfamoyl)-N-(4-(4-chlorothiophen-2-yl)thiazol-2-yl) acetamide (9c) yield 65\% ; m.p.: 154-156º ; IR (KBr): $3366(\mathrm{CONH}), 1715$ (C= O), $1672(\mathrm{C}=\mathrm{C}), 1578(\mathrm{C}=\mathrm{N}), 1342-1146\left(\mathrm{SO}_{2}\right)\left(\mathrm{cm}^{-1}\right)$; ${ }^{1} \mathrm{H}$ NMR $\left(400 \mathrm{MHz}, \mathrm{DMSO}-d_{6}\right): \delta 4.32\left(\mathrm{~s}, 2 \mathrm{H}, \mathrm{CH}_{2}\right), 6.62\left(\mathrm{~s}, 1 \mathrm{H}, \mathrm{C}_{3^{n}}-\mathrm{H}\right), 7.01\left(\mathrm{~s}, 1 \mathrm{H}, \mathrm{C}_{5^{n}}-\mathrm{H}\right), 7.17\left(\mathrm{~s}, 1 \mathrm{H}, \mathrm{C}_{5^{n}}-\mathrm{H}\right), 7.44(\mathrm{~s}$, $\left.1 \mathrm{H}, \mathrm{C}_{3}, \mathrm{H}\right), 7.62-7.87\left(\mathrm{~m}, 5 \mathrm{H}, \mathrm{Ar}-\mathrm{H} \& \mathrm{C}_{5}-\mathrm{H}\right), 10.41$ (bs, $\left.1 \mathrm{H}, \mathrm{CONH}_{2}\right), 10.63$ (bs, $\left.1 \mathrm{H}, \mathrm{NH}\right), 11.78$ (bs,1H, Pyrrole NH) ppm; ${ }^{13} \mathrm{C}$ NMR (100 MHz, DMSO-d $)$ ): $\delta 66.1$ (H $\left.\mathrm{H}_{2} \mathrm{C}-\mathrm{CO}\right), 105.9$ (C-5), 106.6 (C-3"), 117.1 (C-5"), 145.8 (C-4), 146.1 (C-2'), 163.6 (C-2), 171.1 ( C = 0), 121.9, 123.5, 125.3, 126.2, 127.6, 131.3, 134.2, 138.6, 139.4 (Aromatic carbons, C-3', C-4', C-5', C-2" \& C-4") ppm. HRMS (m/z): $536.4152[\mathrm{M}+\mathrm{Na}]^{+}$; Anal. Calcd. for $\mathrm{C}_{19} \mathrm{H}_{14} \mathrm{Cl}_{2} \mathrm{~N}_{4} \mathrm{O}_{3} \mathrm{~S}_{3}: \mathrm{C}, 44.45 ; \mathrm{H}, 2.75 ; \mathrm{N}$, 10.91; Found: C, 44.35; H, 2.72; N, $10.80 \%$.

2-(N-(4-(4-Chlorophenyl)-1H-pyrrol-2-yl)sulfamoyl)-N-(4-(4-nitrothiophen-2-yl)thiazol-2-yl) acetamide (9d) yield 68\% ; m.p.: 162-164 ${ }^{\circ}$; IR (KBr): $3368(\mathrm{CONH}), 1689(\mathrm{C}=\mathrm{O}), 1677(\mathrm{C}=\mathrm{C}), 1572(\mathrm{C}=\mathrm{N}), 1344-1136\left(\mathrm{SO}_{2}\right)\left(\mathrm{cm}^{-1}\right) ;{ }^{1} \mathrm{H}$ $\operatorname{NMR}\left(400 \mathrm{MHz}, \mathrm{DMSO}-d_{6}\right): \delta 4.33\left(\mathrm{~s}, 2 \mathrm{H}, \mathrm{CH}_{2}\right), 6.65\left(\mathrm{~s}, 1 \mathrm{H}, \mathrm{C}_{3^{-}}-\mathrm{H}\right), 7.15\left(\mathrm{~s}, 1 \mathrm{H}, \mathrm{C}_{5^{-}}{ }^{-H}\right), 7.56-7.85\left(\mathrm{~m}, 5 \mathrm{H}, \mathrm{Ar}-\mathrm{H}_{2} \& \mathrm{C}_{5}{ }^{-}\right.$ $\mathrm{H}), 8.49\left(\mathrm{~s}, 1 \mathrm{H}, \mathrm{C}_{5^{-}}-\mathrm{H}\right), 8.82\left(\mathrm{~s}, 1 \mathrm{H}, \mathrm{C}_{3^{-}}-\mathrm{H}\right), 10.31$ (bs, $\left.1 \mathrm{H}, \mathrm{CONH}_{2}\right), 10.60(\mathrm{bs}, 1 \mathrm{H}, \mathrm{NH}), 11.98(\mathrm{bs}, 1 \mathrm{H}, \mathrm{Pyrrole} \mathrm{NH}) \mathrm{ppm}$; ${ }^{13} \mathrm{C}$ NMR (100 MHz, DMSO- $\left.d_{6}\right): \delta 65.6\left(\mathrm{H}_{2} \mathrm{C}-\mathrm{CO}\right), 106.8$ (C-5), 107.9 (C-3"), 116.3 (C-5"), 118.7 (C-3'), 142.3 (C-4), 143.5 (C-2'), 150.1 (C-4'), 164.3 (C-2), 172.8 (C = 0), 124.2, 124.5, 126.3, 129.1, 133.4, 135.6, 137.9 (Aromatic carbons, C-5', C-2" \& C-4") ppm. HRMS (m/z): 546.9713 [M+ Na] ; Anal. Calcd. for $\mathrm{C}_{19} \mathrm{H}_{14} \mathrm{ClN}_{5} \mathrm{O}_{5} \mathrm{~S}_{3}: \mathrm{C}, 43.55 ; \mathrm{H}$, 2.69; N, 13.37; Found: C, 43.48; H, 2.75; N, 13.45\%. 
N-(4-(4-Chlorothiophen-2-yl)thiazol-2-yl)-2-(N-(4-(4-nitrophenyl)-1H-pyrrol-2-yl)sulfamoyl) acetamide (9e) yield 68\% ; m.p.: $155-157^{\circ} \mathrm{C}$; IR (KBr): $3376(\mathrm{CONH}), 1728(\mathrm{C}=0), 1675(\mathrm{C}=\mathrm{C}), 1579(\mathrm{C}=\mathrm{N}), 1338-1145\left(\mathrm{SO}_{2}\right)\left(\mathrm{cm}^{-1}\right) ;{ }^{1} \mathrm{H}$ $\operatorname{NMR}\left(400 \mathrm{MHz}, \mathrm{DMSO}-d_{6}\right): \delta 4.38\left(\mathrm{~s}, 2 \mathrm{H}, \mathrm{CH}_{2}\right), 6.63\left(\mathrm{~s}, 1 \mathrm{H}, \mathrm{C}_{3^{\prime-}} \mathrm{H}\right), 7.10\left(\mathrm{~s}, 1 \mathrm{H}, \mathrm{C}_{5^{\prime \prime}}-\mathrm{H}\right), 7.21\left(\mathrm{~s}, 1 \mathrm{H}, \mathrm{C}_{5^{-1}} \mathrm{H}\right), 7.68-7.84$ $\left(\mathrm{m}, 6 \mathrm{H}, \mathrm{Ar}-\mathrm{H}, \mathrm{C}_{3^{\prime}}-\mathrm{H} \& \mathrm{C}_{5}-\mathrm{H}\right), 10.72(\mathrm{bs}, 1 \mathrm{H}, \mathrm{NH}), 11.08$ (bs, $\left.1 \mathrm{H}, \mathrm{CONH}_{2}\right), 12.06$ (bs,1H, Pyrrole NH) ppm; ${ }^{13} \mathrm{C} \mathrm{NMR}(100$ MHz, DMSO- $\left.d_{6}\right)$ : $\delta 66.3$ ( $\left.\mathrm{H}_{2} \mathrm{C}-\mathrm{CO}\right), 105.8$ (C-5), 107.7 (C-3"), 118.9 (C-5"), 121.8 (C-5'), 123.5 (C-3'), 146.1 (C-4), 150.4 (C-2'), 164.8 (C-2), 171.7 (C= 0), 126.8, 128.4, 130.6, 133.0, 135.3, 136.5, 138.7 (Aromatic carbons, C-4', C-2" \& C-4") ppm. HRMS (m/z): 546.9704 [M + Na] ; Anal. Calcd. for $\mathrm{C}_{19} \mathrm{H}_{14} \mathrm{ClN}_{5} \mathrm{O}_{5} \mathrm{~S}_{3}: \mathrm{C}, 43.55 ; \mathrm{H}, 2.69 ; \mathrm{N}, 13.37$; Found: C, $43.62 ; \mathrm{H}, 2.64 ; \mathrm{N}, 13.30 \%$.

2-(N-(4-(4-Nitrophenyl)-1H-pyrrol-2-yl)sulfamoyl)-N-(4-(4-nitrothiophen-2-yl)thiazol-2-yl) acetamide(9f) yield 70\% ; m.p.: $170-172^{\circ} \mathrm{C}$; IR (KBr): $3374(\mathrm{CONH}), 1714(\mathrm{C}=\mathrm{O}), 1679(\mathrm{C}=\mathrm{C}), 1571(\mathrm{C}=\mathrm{N}), 1335-1139\left(\mathrm{SO}_{2}\right)\left(\mathrm{cm}^{-1}\right) ;{ }^{1} \mathrm{H}$ $\operatorname{NMR}\left(400 \mathrm{MHz}, \mathrm{DMSO}-d_{6}\right): \delta 4.33\left(\mathrm{~s}, 2 \mathrm{H}, \mathrm{CH}_{2}\right), 6.78\left(\mathrm{~s}, 1 \mathrm{H}, \mathrm{C}_{3^{n}}-\mathrm{H}\right), 7.23\left(\mathrm{~s}, 1 \mathrm{H}, \mathrm{C}_{5^{n}}-\mathrm{H}\right), 7.72-7.84\left(\mathrm{~m}, 5 \mathrm{H}, \mathrm{Ar}-\mathrm{H} \& \mathrm{C}_{5}-\mathrm{H}\right)$,

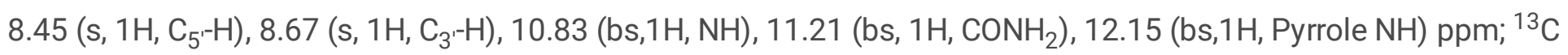
NMR (100 MHz, DMSO-d $)_{6}$ : $\delta 67.6$ (H $\left.\mathrm{H}_{2} \mathrm{C}-\mathrm{CO}\right), 107.2$ (C-5), 108.3 (C-3"), 116.2 (C-5"), 119.2 (C-3'), 151.3 (C-4'), 165.3 (C-2), 172.9 (C = 0), 128.1, 130.4, 132.8, 134.3, 135.4, 136.6, 138.0, 141.2, 142.7 (Aromatic carbons, C-4, C-2', C-5', C2" \& C-4") ppm. HRMS (m/z): $557.5261[\mathrm{M}+\mathrm{Na}]^{+}$; Anal. Calcd. for $\mathrm{C}_{19} \mathrm{H}_{14} \mathrm{~N}_{6} \mathrm{O}_{7} \mathrm{~S}_{3}$ : C, 42.69; $\mathrm{H}, 2.64 ; \mathrm{N}, 15.72 ;$ Found: C, $42.61 ; \mathrm{H}, 2.60 ; \mathrm{N}, 15.64 \%$.

N-(4-(4-Chlorothiophen-2-yl)-1H-imidazol-2-yl)-2-(N-(4-phenyl-1H-pyrrol-2-yl)sulfamoyl) acetamide (10a) yield 69\% ; m.p.: $159-161^{\circ} \mathrm{C}$; IR (KBr): $3364(\mathrm{CONH}), 1668(\mathrm{C}=0), 1653(\mathrm{C}=\mathrm{C}), 1565(\mathrm{C}=\mathrm{N}), 1321-1134\left(\mathrm{SO}_{2}\right)\left(\mathrm{cm}^{-1}\right) ;{ }^{1} \mathrm{H}$ $\operatorname{NMR}\left(400 \mathrm{MHz}, \mathrm{DMSO}-d_{6}\right): \delta 4.26\left(\mathrm{~s}, 2 \mathrm{H}, \mathrm{CH}_{2}\right), 6.57\left(\mathrm{~s}, 1 \mathrm{H}, \mathrm{C}_{3^{\prime \prime}}-\mathrm{H}\right), 7.02\left(\mathrm{~s}, 1 \mathrm{H}, \mathrm{C}_{5^{\prime \prime}}-\mathrm{H}\right), 7.25-7.60\left(\mathrm{~m}, 7 \mathrm{H}, \mathrm{Ar}-\mathrm{H}, \mathrm{C}_{3^{\prime}}-\mathrm{H} \&\right.$ $\left.\mathrm{C}_{5^{-}} \mathrm{H}\right), 7.75\left(\mathrm{~s}, 1 \mathrm{H}, \mathrm{C}_{5}-\mathrm{H}\right), 9.82$ (bs, $\left.1 \mathrm{H}, \mathrm{CONH}_{2}\right), 10.27$ (bs, $\left.1 \mathrm{H}, \mathrm{SO}_{2} \mathrm{NH}\right), 11.40$ (bs, $1 \mathrm{H}$, Pyrrole NH), 12.45 (bs, $1 \mathrm{H}$, Imidazole NH) ppm; ${ }^{13} \mathrm{C}$ NMR (100 MHz, DMSO- $\left.d_{6}\right)$ : $\delta 66.1$ ( $\left.\mathrm{H}_{2} \mathrm{C}-\mathrm{CO}\right), 105.2$ (C-3"), 117.5 (C-5"), 119.1 (C-5), 144.2 (C-2'), 153.2 (C-2), 169.6 (C = O), 122.8, 125.3, 126.7, 127.4, 128.6, 130.2, 132.1, 134.7, 136.8, 139.3 (Aromatic carbons, C-4, C-3', C-4', C-5', C-2" \& C-4") ppm. HRMS (m/z): $484.9291\left[\mathrm{M}+\mathrm{Na}^{+}\right.$; Anal. Calcd. for $\mathrm{C}_{19} \mathrm{H}_{16} \mathrm{ClN}_{5} \mathrm{O}_{3} \mathrm{~S}_{2}: \mathrm{C}_{\text {, }}$ 49.40; H, 3.49; N, 15.16; Found: C, 49.34; H, 3.55; N, $15.24 \%$.

N-(4-(4-Nitrothiophen-2-yl)-7H-imidazol-2-yl)-2-(N-(4-phenyl-1H-pyrrol-2-yl)sulfamoyl) acetamide (10b) yield 70\% ; m.p.: 174-176 C; IR (KBr): $3365(\mathrm{CONH}), 1698(\mathrm{C}=0), 1642(\mathrm{C}=\mathrm{C}), 1568(\mathrm{C}=\mathrm{N}), 1328-1136\left(\mathrm{SO}_{2}\right)\left(\mathrm{cm}^{-1}\right) ;{ }^{1} \mathrm{H}$ NMR (400 MHz, DMSO-d $\left.)_{6}\right): \delta 4.25\left(\mathrm{~s}, 2 \mathrm{H}, \mathrm{CH}_{2}\right), 6.55\left(\mathrm{~s}, 1 \mathrm{H}, \mathrm{C}_{3^{n}}-\mathrm{H}\right), 7.06\left(\mathrm{~s}, 1 \mathrm{H}, \mathrm{C}_{5^{n}}-\mathrm{H}\right), 7.35-7.67(\mathrm{~m}, 5 \mathrm{H}, \mathrm{Ar}-\mathrm{H}), 7.83$ $\left(\mathrm{s}, 1 \mathrm{H}, \mathrm{C}_{5}-\mathrm{H}\right), 8.41\left(\mathrm{~s}, 1 \mathrm{H}, \mathrm{C}_{5^{-}}-\mathrm{H}\right), 8.80\left(\mathrm{~s}, 1 \mathrm{H}, \mathrm{C}_{3^{-}}-\mathrm{H}\right), 10.28$ (bs, $\left.1 \mathrm{H}, \mathrm{CONH}_{2}\right), 10.60$ (bs, 1H, NH), 11.46 (bs, $1 \mathrm{H}$, Pyrrole $\mathrm{NH}), 12.53$ (bs, $1 \mathrm{H}$, Imidazole NH) ppm; ${ }^{13} \mathrm{C}$ NMR (100 MHz, DMSO-d 6 ): $\delta 64.9\left(\mathrm{H}_{2} \mathrm{C}-\mathrm{CO}\right), 104.2$ (C-3"), $116.4(\mathrm{C}-5 ")$, 118.3 (C-3'), 120.2 (C-5), 144.1 (C-2'), 146.7 (C-4'), 153.5 (C-2), 171.9 (C= 0), 125.6, 126.9, 129.3, 131.6, 133.2, 136.5, 138.3, 139.1 (C-4, C-5', C-2", C-4" \& Aromatic carbons) ppm. HRMS (m/z): 495.4843 [M + Na] $]^{+}$; Anal. Calcd. for $\mathrm{C}_{19} \mathrm{H}_{16} \mathrm{~N}_{6} \mathrm{O}_{5} \mathrm{~S}_{2}: 48.30 ; \mathrm{H}, 3.41 ; \mathrm{N}, 17.79$; Found: $\mathrm{C}, \mathrm{C}, 48.36 ; \mathrm{H}, 3.47 ; \mathrm{N}, 17.89 \%$.

2-(N-(4-(4-Chlorophenyl)-1H-pyrrol-2-yl)sulfamoyl)-N-(4-(4-chlorothiophen-2-yl)-1H-imidazol-2-yl)acetamide (10c) yield 69\% ; m.p.: $185-187^{\circ} \mathrm{C}$; IR (KBr): $3362(\mathrm{CONH}), 1676(\mathrm{C}=0), 1668(\mathrm{C}=\mathrm{C}), 1571(\mathrm{C}=\mathrm{N}), 1329-1125\left(\mathrm{SO}_{2}\right)$ $\left(\mathrm{cm}^{-1}\right) ;{ }^{1} \mathrm{H}$ NMR $\left(400 \mathrm{MHz}, \mathrm{DMSO}-d_{6}\right): \delta 4.28\left(\mathrm{~s}, 2 \mathrm{H}, \mathrm{CH}_{2}\right), 6.54\left(\mathrm{~s}, 1 \mathrm{H}, \mathrm{C}_{3^{\prime \prime}} \mathrm{H}\right), 7.05\left(\mathrm{~s}, 1 \mathrm{H}, \mathrm{C}_{5^{-1}} \mathrm{H}\right), 7.18\left(\mathrm{~s}, 1 \mathrm{H}, \mathrm{C}_{5^{-}}-\mathrm{H}\right)$, 7.55-7.80 (m, 6H, Ar-H, $\left.\mathrm{C}_{3^{2}}-\mathrm{H} \& \mathrm{C}_{5}-\mathrm{H}\right), 10.17$ (bs, 1H, CONH 2$), 10.28$ (bs, 1H, NH), 11.89 (bs, 1H, Pyrrole NH) 12.60 (bs,1H, Imidazole NH) ppm; ${ }^{13} \mathrm{C}$ NMR (100 MHz, DMSO-d 6 ): $\delta 64.8$ (H $\left.\mathrm{H}_{2} \mathrm{C}-\mathrm{CO}\right), 106.1$ (C-3"), 117.5 (C-5"), 119.2 (C-5), 144.1 (C-2'), 153.8 (C-2), 170.1 (C = 0), 122.2, 124.6, 126.7, 128.5, 130.5, 133.6, 135.4, 136.3, 137.1, 138.7 (Aromatic 
carbons, C-4, C-3', C-4', C-5', C-2" \& C-4") ppm. HRMS (m/z): 519.3705 [M + Na]+; Anal. Calcd. for $\mathrm{C}_{19} \mathrm{H}_{15} \mathrm{Cl}_{2} \mathrm{~N}_{5} \mathrm{O}_{3} \mathrm{~S}_{2}: \mathrm{C}, 45.97 ; \mathrm{H}, 3.05 ; \mathrm{N}, 14.11$; Found: $\mathrm{C}, 45.90 ; \mathrm{H}, 3.00 ; \mathrm{N}, 14.23 \%$.

2-(N-(4-(4-Chlorophenyl)-1H-pyrrol-2-yl)sulfamoyl)-N-(4-(4-nitrothiophen-2-yl)-1H-imidazol-2-yl)acetamide (10d) yield 69\% ; m.p.: 194-196º C; IR (KBr): 3361 (CONH), 1695 (C= O), $1637(\mathrm{C}=\mathrm{C}), 1570(\mathrm{C}=\mathrm{N}), 1331-1136\left(\mathrm{SO}_{2}\right)$ $\left(\mathrm{cm}^{-1}\right) ;{ }^{1} \mathrm{H}$ NMR $\left(400 \mathrm{MHz}, \mathrm{DMSO}-d_{6}\right): \delta 4.27\left(\mathrm{~s}, 2 \mathrm{H}, \mathrm{CH}_{2}\right), 6.58\left(\mathrm{~s}, 1 \mathrm{H}, \mathrm{C}_{3^{n}} \mathrm{H}\right), 7.09\left(\mathrm{~s}, 1 \mathrm{H}, \mathrm{C}_{5^{n}} \mathrm{H}\right), 7.70-7.88(\mathrm{~m}, 5 \mathrm{H}$, Ar- $\left.-\mathrm{H} \& \mathrm{C}_{5}-\mathrm{H}\right), 8.41\left(\mathrm{~s}, 1 \mathrm{H}, \mathrm{C}_{5}-\mathrm{H}\right), 8.78\left(\mathrm{~s}, 1 \mathrm{H}, \mathrm{C}_{3^{\prime}}-\mathrm{H}\right), 10.38$ (bs, 1H, CONH $\left.)_{2}\right), 10.56$ (bs, 1H, NH), 11.86 (bs, $1 \mathrm{H}$, Pyrrole $\mathrm{NH}), 12.59$ (bs, $1 \mathrm{H}$, Imidazole NH) ppm; ${ }^{13} \mathrm{C}$ NMR (100 MHz, DMSO- $\left.d_{6}\right): \delta 64.2\left(\mathrm{H}_{2} \mathrm{C}-\mathrm{CO}\right), 106.8$ (C-3"), 116.2 (C-5"), 117.9 (C-3'), 119.5 (C-5), 144.6 (C-2'), 149.5 (C-4'), 153.6 (C-2), 170.2 (C = 0), 123.7, 126.1, 128.9, 130.3, 134.2, 136.2, 137.1, 139.3 (Aromatic carbons, C-4, C-5', C-2" \& C-4") ppm. HRMS (m/z): 529.9264 [M + Na] $]^{+}$; Anal. Calcd. for $\mathrm{C}_{19} \mathrm{H}_{15} \mathrm{ClN}_{6} \mathrm{O}_{5} \mathrm{~S}_{2}$ : C, 45.02; $\mathrm{H}, 2.98 ; \mathrm{N}, 16.58$; Found: $\mathrm{C}, 44.96 ; \mathrm{H}, 2.91 ; \mathrm{N}, 16.65 \%$.

N-(4-(4-Chlorothiophen-2-yl)-1H-imidazol-2-yl)-2-(N-(4-(4-nitrophenyl)-1H-pyrrol-2-yl) sulfamoyl)acetamide(10e) yield 72\% ; m.p.: 183-185² C; IR (KBr): 3369 (CONH), 1718 (C= O), $1643(\mathrm{C}=\mathrm{C}), 1580(\mathrm{C}=\mathrm{N}), 1341-1138\left(\mathrm{SO}_{2}\right)$ $\left(\mathrm{cm}^{-1}\right) ;{ }^{1} \mathrm{H}$ NMR $\left(400 \mathrm{MHz}, \mathrm{DMSO}-d_{6}\right): \delta 4.35\left(\mathrm{~s}, 2 \mathrm{H}, \mathrm{CH}_{2}\right), 6.68\left(\mathrm{~s}, 1 \mathrm{H}, \mathrm{C}_{3^{n-}} \mathrm{H}\right), 7.07\left(\mathrm{~s}, 1 \mathrm{H}, \mathrm{C}_{5^{n}} \mathrm{H}\right), 7.16\left(\mathrm{~s}, 1 \mathrm{H}, \mathrm{C}_{5^{-}} \mathrm{H}\right)$, $7.42\left(\mathrm{~s}, 1 \mathrm{H}, \mathrm{C}_{3^{2}-\mathrm{H}}\right), 7.56-7.78\left(\mathrm{~m}, 5 \mathrm{H}, \mathrm{Ar}-\mathrm{H} \& \mathrm{C}_{5}-\mathrm{H}\right), 10.49$ (bs, 1H, CONH $)_{2}, 10.67$ (bs, $\left.1 \mathrm{H}, \mathrm{NH}\right), 11.96$ (bs, $1 \mathrm{H}$, Pyrrole $\mathrm{NH}), 12.65$ (bs, $1 \mathrm{H}$, Imidazole NH) ppm; ${ }^{13} \mathrm{C}$ NMR (100 MHz, DMSO- $\left.d_{6}\right): \delta 65.5\left(\mathrm{H}_{2} \mathrm{C}-\mathrm{CO}\right), 106.6$ (C-3"), 117.8 (C-5"), 145.8 (C-2'), 151.9 (C-2), 171.6 (C = 0), 121.3, 124.6, 126.4, 127.1, 129.5, 131.1, 132.4, 133.6, 134.5, 135.9, 137.8 (Aromatic carbons, C-4, C-5, C-3', C-4', C-5', C-2" \& C-4") ppm. HRMS (m/z): 529.9255 [M + Na] $]^{+}$; Anal. Calcd. for $\mathrm{C}_{19} \mathrm{H}_{15} \mathrm{ClN}_{6} \mathrm{O}_{5} \mathrm{~S}_{2}$ : C, 45.02; $\mathrm{H}, 2.98 ; \mathrm{N}, 16.58$; Found: $\mathrm{C}, 44.95 ; \mathrm{H}, 3.03 ; \mathrm{N}, 16.67 \%$.

2-(N-(4-(4-Nitrophenyl)-1H-pyrrol-2-yl)sulfamoyl)-N-(4-(4-nitrothiophen-2-yl)-1H-imidazol-2-yl) acetamide (10f) yield 73\% ; m.p.: 210-212 ${ }^{\circ} \mathrm{C}$; IR (KBr): $3378(\mathrm{CONH}), 1728$ (C= )), $1667(\mathrm{C}=\mathrm{C}), 1565(\mathrm{C}=\mathrm{N}), 1329-1141\left(\mathrm{SO}_{2}\right)\left(\mathrm{cm}^{-1}\right)$;

${ }^{1} \mathrm{H}$ NMR $\left(400 \mathrm{MHz}, \mathrm{DMSO}-d_{6}\right): \delta 4.21\left(\mathrm{~s}, 2 \mathrm{H}, \mathrm{CH}_{2}\right), 6.57\left(\mathrm{~s}, 1 \mathrm{H}, \mathrm{C}_{3^{n}} \mathrm{H}\right), 7.10\left(\mathrm{~s}, 1 \mathrm{H}, \mathrm{C}_{5^{n}} \mathrm{H}\right), 7.58-7.72(\mathrm{~m}, 5 \mathrm{H}, \mathrm{Ar}-\mathrm{H}$ \& $\left.\mathrm{C}_{5}-\mathrm{H}\right), 8.28\left(\mathrm{~s}, 1 \mathrm{H}, \mathrm{C}_{5}-\mathrm{H}\right), 8.73\left(\mathrm{~s}, 1 \mathrm{H}, \mathrm{C}_{3^{\prime}}-\mathrm{H}\right), 10.43\left(\mathrm{bs}, 1 \mathrm{H}, \mathrm{CONH}_{2}\right), 10.60$ (bs,1H, NH), 11.48 (bs,1H, Pyrrole NH), 12.54 (bs,1H, Imidazole NH) ppm; ${ }^{13} \mathrm{C}$ NMR (100 MHz, DMSO- $\left.d_{6}\right): \delta 66.2\left(\mathrm{H}_{2} \mathrm{C}-\mathrm{CO}\right), 107.5(\mathrm{C}-3 "), 116.2(\mathrm{C}-5 "), 118.3$ (C-3'), 121.5 (C-5), 149.8 (C-4'), 152.7 (C-2), 171.9 (C = 0), 126.9, 128.4, 130.7, 133.7, 135.4, 137.8, 140.4, 142.6, 145.0 (Aromatic carbons, C-4, C-2', C-5', C-2" \& C-4") ppm. HRMS (m/z): 540.4803 [M + Na] $]^{+}$; Anal. Calcd. for $\mathrm{C}_{19} \mathrm{H}_{15} \mathrm{~N}_{7} \mathrm{O}_{7} \mathrm{~S}_{2}$ : C, 44.10; H, 2.92; N, 18.95; Found: C, 44.03; H, 2.97; N, $18.83 \%$.

\section{Biological Activity Assays}

The in vitro antimicrobial studies were determined by agar well diffusion method against test organisms [Mounyr et al. 2016]. Nutrient broth (NB) plates were swabbed with $24 \mathrm{~h}$ old broth culture $(100 \mu \mathrm{L})$ of test bacteria. Wells $(6$ $\mathrm{mm}$ ) were made into each petriplate using the sterile cork borer. The compounds were dissolved in DMSO (5 $\mathrm{mg} / \mathrm{mL})$ and from this $2.5,5,10$, and $20 \mu \mathrm{L}(12.5,25,50,100 \mu \mathrm{g} /$ well) were added into the wells by using sterile pipettes. The standard antibiotics (positive control), chloramphenicol for antibacterial activity and ketoconazole for antifungal activity were tested against the pathogens simultaneously. The samples were dissolved in DMSO which showed no zone of inhibition acts as negative control. The plates were incubated at $37^{\circ} \mathrm{C}$ for $24 \mathrm{~h}$ for bacteria and at $28{ }^{\circ} \mathrm{C}$ for $48 \mathrm{~h}$ for fungi. The diameter of zone of inhibition of each well was measured after appropriate incubation. Duplicates were done and the average values were calculated for eventual antibacterial activity. Broth dilution test was used to determine minimum inhibitory concentration (MIC) of the above samples 
[Bishnu et al. 2009]. Freshly prepared nutrient broth was used as diluents. The $24 \mathrm{~h}$ old culture of the test bacteria Staphylococcus aureus, Bacillus subtilis, Pseudomonas aeruginosa and Klebsiella pneumoniae and the test fungi Aspergillus niger and Penicillium chrysogenum were diluted 100 folds in nutrient broth (100 $\mu \mathrm{L}$ bacterial cultures in $10 \mathrm{~mL} \mathrm{NB}$ ). The stock solution of the compounds was prepared in DMSO by dissolving $5 \mathrm{mg}$ of the compound in 1 $\mathrm{mL}$ of DMSO. Increasing concentrations of the test samples $(2.5,5,10,20 \mu \mathrm{L}$ of stock solution contains $12.5,25$, $50,100 \mu \mathrm{g}$ of the compounds) were added to the test tubes containing the bacterial and fungal cultures. All the tubes were incubated at $37^{\circ} \mathrm{C}$ for $24 \mathrm{~h}$ for bacteria and at $28^{\circ} \mathrm{C}$ for $48 \mathrm{~h}$ for fungi. The tubes were examined for visible turbidity and using NB as control. Simultaneously control without test samples and with solvent was assayed. The MIC was recorded as the lowest concentration that inhibited visible growth of the tested organisms. To determine the minimum bactericidal concentration (MBC) [Pennsylvania et al. 2006] and minimum fungicidal concentration (MFC) [Pennsylvania et al. 2002] for each set of test tubes in the MIC determination, a loopful of broth was collected from those tubes which did not show any growth and inoculated on sterile nutrient broth (for bacteria) and PDA (for fungi) by streaking. These inoculated plates were incubated at $37^{\circ} \mathrm{C}$ for $24 \mathrm{~h}$ (bacteria) and at $28{ }^{\circ} \mathrm{C}$ for $48 \mathrm{~h}$ (fungi). After incubation, the lowest concentration was noted as MBC or MFC at which no visible growth was observed.

\section{Declarations}

\section{Acknowledgements}

The authors are grateful to CSIR (Council of Scientific and Industrial Research), New Delhi for financial assistance under major research project.

\section{References}

1. Ahmed K, Naseer M, Srinivasa Reddy AK, Rohini K K (2007) Synthesis of a new class of 2- anilino substituted nicotinyl arylsulfonylhydrazides as potential anticancer and antibacterial agents. Bioorg Med Chem 15:10041013

2. Andrea T, Roberta C, Maurizio B, Roberto DS, Federico C, Silvio M, Andrea C, Fabrizio M, Marino A (2002) Antifungal Agents. 10. New Derivatives of 1-[(Aryl)[4-aryl-1H-pyrrol-3-yl]methyl]-1H-imidazole, Synthesis, AntiCandida Activity, and Quantitative Structure-Analysis Relationship Studies. J Med Chem 45:2720-2732

3. Ahmad N, Zia-ur-Rehman M, Latif Siddiqui H, Fasih Ullah M, Parvez M (2011) Microwave assisted synthesis and structureeactivity relationship of 4-hydroxy-N0-[1- phenylethylidene]-2H/2-methyl-1,2-benzothiazine-3carbohydrazide-1,1-dioxides as anti- microbial agents. Eur J Med Chem 46:2368-2377

4. Bishnu j, Sunil L, Anuja S (2009) Antibacterial Property of Different Medicinal Plants: Ocimum sanctum, Cinnamomum zeylanicum, Xanthoxylum armatum and Origanum majorana

5. kathmandu university journal of science, engineering and technology 5:143-150

6. Chandrasekhar S, Sudhakar A (2010) Total Synthesis of Bengazole A. Org Lett 12:2

7. Debasish B, Sanghamitra M, Jose CG, John DS, Bimal KB (2012) Ultrasound-assisted bismuth nitrate-induced green synthesis of novel pyrrole derivatives and their biological evaluation as anticancer agents. Eur $\mathrm{J}$ Med Chem 50:209-215

8. Divya K, Sravya G, Padmaja A, Padmavathi V (2015) Synthesis and antimicrobial activity of bis-heterocyclic sulfamoyl acetamides. Res Chem Intermed 41:4413 
9. Erhorn SP, Pharm X (2007) The Comprehensive Pharmacology Reference. 1-5 (b) Kulikowska J, Celinski R, Soja A, Sybirska H (2002) Problems of Forensic Sciences. XLIX. Cracow, Poland, Institute of Forensic Research, 99

10. Huihua Sun H, Zhang EL, Ang H, Zhao (2018) Biocatalysis for the synthesis of pharmaceuticals and pharmaceutical intermediates. Bioorg. Med. Chem. Lett 26:1275- 1284 (b) Honey, Suresh T, Manoj K, Sinha VR (2012) Self-organizing molecular field analysis of NSAIDs: Assessment of pharmacokinetic and physicochemical properties using 3D-QSPkR approach. Eur. J. Med. Chem 53:76-82

11. Harrak Y, Rosell G, Daidone G, Plescia S, Schillacib D, Pujola MD (2007) Synthesis and biological activity of new anti-inflammatory compounds containing the 1,4-benzodioxine and/or pyrrole system. Bioorg Med Chem Lett 15:4876-4890

12. Hudson JB, Graham EA, Micki hudson, Towers GHN (1986) antiviral activity of the photoactive thiophene aterthienyl. Photochem Photobiol 44:4:477-482

13. Ibrahim Evren K, Mustafa S, Ertan SA, Ismail Y (2017) Preparation of novel pyrrol-2-one derivatives via an effective synthesis of new oxazole scaffold. Tetrahedron 73:1795-1802

14. Jacques L, Bernard F, Laurence B, Marina O, Alain P, Jean-Michel V (1999) Synthesis and antioxidant activity of new tetraarylpyrroles. Eur J Med Chem 34:991-996

15. John J, Koshy SKGJ (2012) Am Board Fam Med 25:343-349

16. Karl JH, Milosz G, Soraya M, Maciej M (2014) Asymmetric Total Synthesis of (+)-Inthomycin C via O-Directed Free Radical Alkyne Hydrostannation with $\mathrm{Ph}_{3} \mathrm{SnH}$ and Catalytic $\mathrm{Et}_{3} \mathrm{~B}$ : Reinstatement of the Zeeck - Taylor (3R)-Structure for (+)-Inthomycin C. Org Lett 16:1164-1167

17. Kristina S, Marijeta K, Katja E, Ivan S, Magdalena G, Kresimir P, Karminski-Zamolaa G (2007) Synthesis, antiviral and antitumor activity of 2-substituted-5-amidino-benzimidazoles. Bioorg Med Chem Lett 15:44194426

18. Kristin EP, Claude Larrivee A, Brett ML, McLaughlin RW, Jason M, Kevin WH, Joel MH

19. Rajappa V (2009) Mild and Efficient DBU-Catalyzed Amidation of Cyanoacetates. Org Lett 11:9

20. Leyla Y, Murat D, Demirayak S, Karaca Gencer H, Yagmur T (2013) Synthesis and initial biological evaluation of substituted 1-phenylamino-2-thio-4,5-dimethyl-1H-imidazole derivatives. Bioorg Med Chem Lett 23:67646768

21. Mark CW, Patrick B, Tim L (2011) Lipophilicity of acidic compounds: Impact of ion pair partitioning on drug design. Bioorg Med Chem Lett 21:3550-3556

22. Ming-Zhong Han X, Tuan-Wei L, Qi F, Shu-Jing Y, Su-Hua W, Zheng-Ming L (2011) Design, synthesis and antifungal activities of novel pyrrole alkaloid analogs. Eur J Med Chem 46:1463-1472

23. Marcocci L, Maguire JJ, Droy-Lefaix MT, Packer L (1994) Biochem Biophys Res Commun 201:748

24. Mounyr B, MoulayS, Saad Koraichi I (2016) Methods for in vitro evaluating antimicrobial activity. Journal of Pharmaceutical Analysis 671-79

25. Przybilla B, Schwab-Przybilla U, Ruzicka T, Ring J (1987) Photodermatology 4:73-78

26. Pennsylvania (2006) Methods for dilution antimicrobial susceptibility tests for bacteria that grow aerobically, 7th edition, approved standard, CLSI Document M7-A7 Ways. Clinical and Laboratory Standards Institute

27. Pennsylvania (2002) Method for broth dilution antifungal susceptibility testing of yeasts, approved standard NCCLS Document M27-A2, Ways. Clinical and Laboratory Standards 
28. Institute

29. Pasha AM, Khan R, Shrivasta N Ultra sonochem 26(15) 2015

30. Premakumari C, Seenaiah D, Srilakshmi A, Venkata Ramana D, Sai Gopal, Padmavathi V

31. Heterocyclic J chem 51:1875

32. Ritesh NS, Franklin PX, Kamala KV, Subhash CC, Shyam SP (2009) Synthesis of 4-benzyl-1,3-thiazole derivatives as potential anti-inflammatory agents: An analogue-based drug design approach. J Enzyme Inhib Med Chem 24(3):890-897

33. Ramandeep K, Kapil K (2018) One-pot synthesis of [4-(tert-butyl)-1H-pyrrol-3-yl](phenyl)methanone from tosylmethyl isocyanide and carbonyl compound. Chem Heterocycl Compd 54(7):700-702

34. Roland W. Bu R, Peter J, Dustin M, Quan L, Jian-Xin D, Jacob AK, Stacey D, Heinz EM (2004) DNA binding ligands targeting drug-resistant Gram-positive bacteria. Part 2: C-terminal benzimidazoles and derivatives. Bioorg Med Chem Lett 14:1259-1263

35. Ronald AF, Joel E. Wright Andre R (2002) Synthesis and In Vitro Antitumor Activity of Thiophene Analogues of 5-Chloro-5,8-dideazafolic Acid and 2-Methyl-2-desamino-5- chloro-5,8-dideazafolic Acid. Bioorg Med Chem Lett 10:2067-2076

36. Rekha T, Durgamma S, Padmaja A, Padmavathi V (2017) Synthesis and antimicrobial activity of bis(azolyl)quinazoline-2,4-diamines. Monatsh Chem 148:1781

37. Samir B, Tamer N, Yousry AA (2013) Synthesis of some new 2-(3-pyridyl)-4,5-disubstituted

38. thiazoles as potent antimicrobial agents. Eur. J. Med. Chem 62:270-279

39. Samir B, Walid F, Mohamed AM (2010) Synthesis and antimicrobial activity of some new thiazole, thiophene and pyrazole derivatives containing benzothiazole moiety. Eur J Med Chem 45:3692-3701

40. Siva Sankar P, Rekha T, Padmaja A, Padmavathi V, Siva Krishna N, Kondaiah P (2019) Synthesis, cytotoxic and antioxidant activities of azolyl benzothiazine. carboxamides Res Chem Intermed 45:3053-3075

41. Sowmya DV, Lakshmi Teja G, Padmaja A, Kamala Prasad V, Padmavathi V (2018) Green approach for the synthesis of thiophenyl pyrazoles and isoxazoles by adopting 1,3-dipolar cycloaddition methodology and their antimicrobial activity. Eur J Med Chem 143:891-898

42. Sabot C, Ananda Kumar K, Meunier S, Mioskowski C (2007) A convenient aminolysis of esters catalyzed by 1,5,7- triazabicyclo[4.4.0]dec-5-ene (TBD) under solvent-free conditions. Tetrahedron Lett 48:3863-3866

43. Taterao MP, Sachin Al, Kumar VS (2008) Catalyst-free efficient synthesis of 2-aminothiazoles in water at ambient temperature. Tetrahedron 64:5019-5022

44. Thomas LL, Stephen EW (1994) A Simple and Practical Synthesis of 2-Aminoimidazoles. J Org Chem 59:7299-7305

45. Valentin Z, Adriana I, Nicolae P, Bathelemy N, Victor K, Charles NF, Marlyse LM, Bonaventure TN (2010) Synthesis of some p-toluenesulfonyl-hydrazinothiazoles and hydrazino-bisthiazoles and their anticancer activity. Eur J Med Chem 45:5080-5085

46. Wilson KJ, Illig CR, Subasinghe N, Hoffman JB, Jonathan Rudolph M, Soll R, Molloy CJ, Bone R, David G, Troy R, Marie Z, Lewandowski FA, Zhou Z, Sharp C, Maguire D, Grasberger B, DesJarlais RL, Spurlino J (2001) Synthesis of Thiophene-2- carboxamidines Containing 2-Aminothiazoles and their Biological Evaluation as Urokinase Inhibitors. Bioorg Med Chem Lett 11:915-918

47. Xing Y, Vladimir BB (2009) Acyl Transfer Catalysis with 1,2,4-Triazole Anion Org. Lett 11:7 
48. Zia-ur-Rehman M, Anwar Choudary J, James Elsegood MR, Latif Siddiqui H, Mohammad Khan K (2009) A facile synthesis of novel biologically active 4-hydroxy-NO-(benzylidene)-2H- benzo[e][1,2]thiazine-3carbohydrazide 1,1-dioxides. Eur J Med Chem 44:1311-1316

\section{Tables}

Table 1: Reaction of $\mathbf{4 a}$ with $7 \mathbf{a}$ in the presence of different molar concentrations of $\mathrm{NaOMe}$

\begin{tabular}{|lll|}
\hline S. No & mol \% of NaOMe & Yield \\
\hline 1 & $2.0 \mathrm{~mol} \%$ & \\
\hline 2 & $3.0 \mathrm{~mol} \%$ & 48 \\
\hline 3 & $4.0 \mathrm{~mol} \%$ & 54 \\
\hline 4 & $5.0 \mathrm{~mol} \%$ & 78 \\
\hline 5 & $6.0 \mathrm{~mol} \%$ & 62 \\
\hline
\end{tabular}

Table 3. The in vitro antifungal activity of compounds 8-10

$(-)$ No activity. ( \pm ) Standard deviation

Table 4. MIC, MBC and MFC of compounds $9 \mathrm{~d}, 9 \mathrm{e}, 9 \mathrm{f}, 10 \mathrm{~d}, 10 \mathrm{e}$ and $10 \mathrm{f}$

(-) No activity. ( \pm ) Standard deviation.

Due to technical limitations, Table 2 is only available as a download in the Supplemental Files section.

\section{Figures}




\begin{tabular}{|c|c|c|c|c|c|c|c|c|}
\hline \multirow{3}{*}{$\begin{array}{l}\text { Compound } \\
\text { No. }\end{array}$} & \multicolumn{8}{|c|}{ Zone of Inhibition (mm) } \\
\hline & \multicolumn{4}{|l|}{ A. niger } & \multicolumn{4}{|c|}{ P. chrysogenum } \\
\hline & $\begin{array}{l}12.5 \\
\mu \mathrm{g} / \mathrm{well}\end{array}$ & $\begin{array}{l}25 \\
\mu \mathrm{g} / \text { well }\end{array}$ & $\begin{array}{l}50 \\
\mu \mathrm{g} / \text { well }\end{array}$ & $\begin{array}{l}100 \\
\mu g / \text { well }\end{array}$ & $\begin{array}{l}12.5 \\
\mu g / \text { well }\end{array}$ & $\begin{array}{l}25 \\
\mu \mathrm{g} / \text { well }\end{array}$ & $\begin{array}{l}50 \\
\mu g / \text { well }\end{array}$ & $\begin{array}{l}100 \\
\mu g / \text { well }\end{array}$ \\
\hline $8 a$ & $8 \pm 1.6$ & - & $10 \pm 1.2$ & $12 \pm 2.1$ & - & - & $9 \pm 1.1$ & $11 \pm 2.2$ \\
\hline $8 b$ & - & $9 \pm 1.7$ & $11 \pm 1.5$ & $14 \pm 1.9$ & - & - & $11 \pm 2.0$ & $13 \pm 1.4$ \\
\hline $8 c$ & $10 \pm 1.1$ & $12 \pm 2.5$ & $13 \pm 2.0$ & $15 \pm 1.3$ & - & $9 \pm 1.2$ & $12 \pm 1.5$ & $15 \pm 2.0$ \\
\hline $8 d$ & $12 \pm 2.0$ & $13 \pm 2.1$ & $17 \pm 2.4$ & $21 \pm 2.2$ & $10 \pm 1.4$ & $12 \pm 1.6$ & $13 \pm 1.7$ & $15 \pm 1.9$ \\
\hline $8 e$ & $13 \pm 1.7$ & $15 \pm 2.0$ & $18 \pm 2.2$ & $20 \pm 1.9$ & $11 \pm 1.2$ & $13 \pm 1.8$ & $14 \pm 2.0$ & $16 \pm 2.6$ \\
\hline $8 f$ & $19 \pm 2.3$ & $22 \pm 2.4$ & $25 \pm 2.6$ & $28 \pm 2.8$ & $15 \pm 2.0$ & $18 \pm 1.9$ & $20 \pm 2.3$ & $22 \pm 2.5$ \\
\hline $9 a$ & $12 \pm 2.6$ & $14 \pm 2.7$ & $16 \pm 2.0$ & $17 \pm 1.3$ & $9 \pm 2.2$ & $11 \pm 2.4$ & $14 \pm 2.5$ & $16 \pm 2.8$ \\
\hline $9 b$ & $23 \pm 2.2$ & $25 \pm 2.3$ & $28 \pm 1.8$ & $31 \pm 1.2$ & $15 \pm 1.7$ & $17 \pm 1.3$ & $19 \pm 1.8$ & $22 \pm 1.4$ \\
\hline $9 c$ & $21 \pm 1.8$ & $25 \pm 1.2$ & $27 \pm 1.4$ & $29 \pm 2.0$ & $17 \pm 1.5$ & $18 \pm 2.2$ & $21 \pm 2.4$ & $24 \pm 1.3$ \\
\hline $9 d$ & $27 \pm 2.1$ & $29 \pm 2.5$ & $31 \pm 2.8$ & $33 \pm 2.7$ & $19 \pm 2.5$ & $22 \pm 2.9$ & $25 \pm 2.6$ & $27 \pm 2.7$ \\
\hline $9 e$ & $26 \pm 2.3$ & $29 \pm 2.2$ & $30 \pm 1.9$ & $32 \pm 2.4$ & $21 \pm 2.8$ & $23 \pm 2.6$ & $26 \pm 1.3$ & $28 \pm 2.2$ \\
\hline $9 f$ & $31 \pm 2.7$ & $33 \pm 1.5$ & $34 \pm 2.3$ & $37 \pm 1.7$ & $25 \pm 2.6$ & $27 \pm 2.8$ & $29 \pm 1.5$ & $31 \pm 2.4$ \\
\hline $10 a$ & $20 \pm 1.6$ & $23 \pm 1.3$ & $25 \pm 1.6$ & $26 \pm 2.7$ & $13 \pm 2.4$ & $15 \pm 2.5$ & $17 \pm 2.1$ & $20 \pm 1.0$ \\
\hline $10 \mathrm{~b}$ & $26 \pm 2.4$ & $27 \pm 2.6$ & $29 \pm 2.1$ & $32 \pm 1.2$ & $18 \pm 1.8$ & $20 \pm 2.8$ & $23 \pm 1.5$ & $25 \pm 2.7$ \\
\hline $10 c$ & $27 \pm 2.1$ & $29 \pm 2.3$ & $30 \pm 1.7$ & $33 \pm 1.5$ & $21 \pm 1.6$ & $24 \pm 1.5$ & $26 \pm 1.6$ & $28 \pm 1.2$ \\
\hline $10 d$ & $31 \pm 2.3$ & $34 \pm 1.7$ & $35 \pm 2.5$ & $38 \pm 1.4$ & $22 \pm 2.8$ & $24 \pm 2.7$ & $27 \pm 1.9$ & $30 \pm 2.3$ \\
\hline $10 e$ & $30 \pm 2.1$ & $32 \pm 1.8$ & $35 \pm 2.2$ & $38 \pm 1.8$ & $23 \pm 2.3$ & $25 \pm 2.5$ & $28 \pm 1.4$ & $30 \pm 2.1$ \\
\hline $10 f$ & $34 \pm 2.5$ & $36 \pm 2.4$ & $39 \pm 2.7$ & $41 \pm 1.6$ & $26 \pm 2.9$ & $27 \pm 2.3$ & $30 \pm 1.7$ & $32 \pm 2.8$ \\
\hline Ketoconazole & $29 \pm 1.4$ & $31 \pm 2.2$ & $33 \pm 1.6$ & $36 \pm 2.1$ & $33 \pm 3.2$ & $35 \pm 1.7$ & $36 \pm 2.5$ & $38 \pm 1.4$ \\
\hline $\begin{array}{l}\text { Control } \\
\text { (DMSO) }\end{array}$ & - & - & - & - & - & - & - & - \\
\hline
\end{tabular}




\section{MIC (MBC/MFC) mg/well}

\section{$\begin{array}{lllll}\text { S. aureus B. subtilis } & \underset{P}{P} & \text { K.pneumoniae } & \text { A.niger } & \text { P.chrysogenum }\end{array}$}

\begin{tabular}{lllllll}
\hline 9d & $12.5(50)$ & $6.25(12.5)$ & $12.5(50)$ & $100(>200)$ & $12.5(50)$ & $200(-)$ \\
\hline $9 \mathbf{e}$ & $12.5(50)$ & $6.25(12.5)$ & $12.5(50)$ & $100(>200)$ & $12.5(50)$ & $200(-)$ \\
\hline $\mathbf{9 f}$ & $6.25(12.5)$ & $6.25(12.5)$ & $12.5(50)$ & $50(200)$ & $6.25(12.5)$ & $100(>200)$ \\
\hline $\mathbf{1 0 d}$ & $50(200)$ & $12.5(100)$ & $50(200)$ & $200(-)$ & $6.25(12.5)$ & $100(>200)$ \\
$10 \mathbf{e}$ & $50(200)$ & $12.5(100)$ & $50(200)$ & $200(-)$ & $6.25(12.5)$ & $100(>200)$ \\
$\mathbf{1 0 f}$ & $25(100)$ & $6.25(12.5)$ & $50(200)$ & $200(-)$ & .2 & $100(>200)$ \\
\hline C & & & & & $6.25(12.5)$ & - \\
Chloramphenicol & 6.25 & 6.25 & 6.25 & 12.5 & - & - \\
\hline Ketoconazole & - & - & - & - & 6.25 & 12.5
\end{tabular}

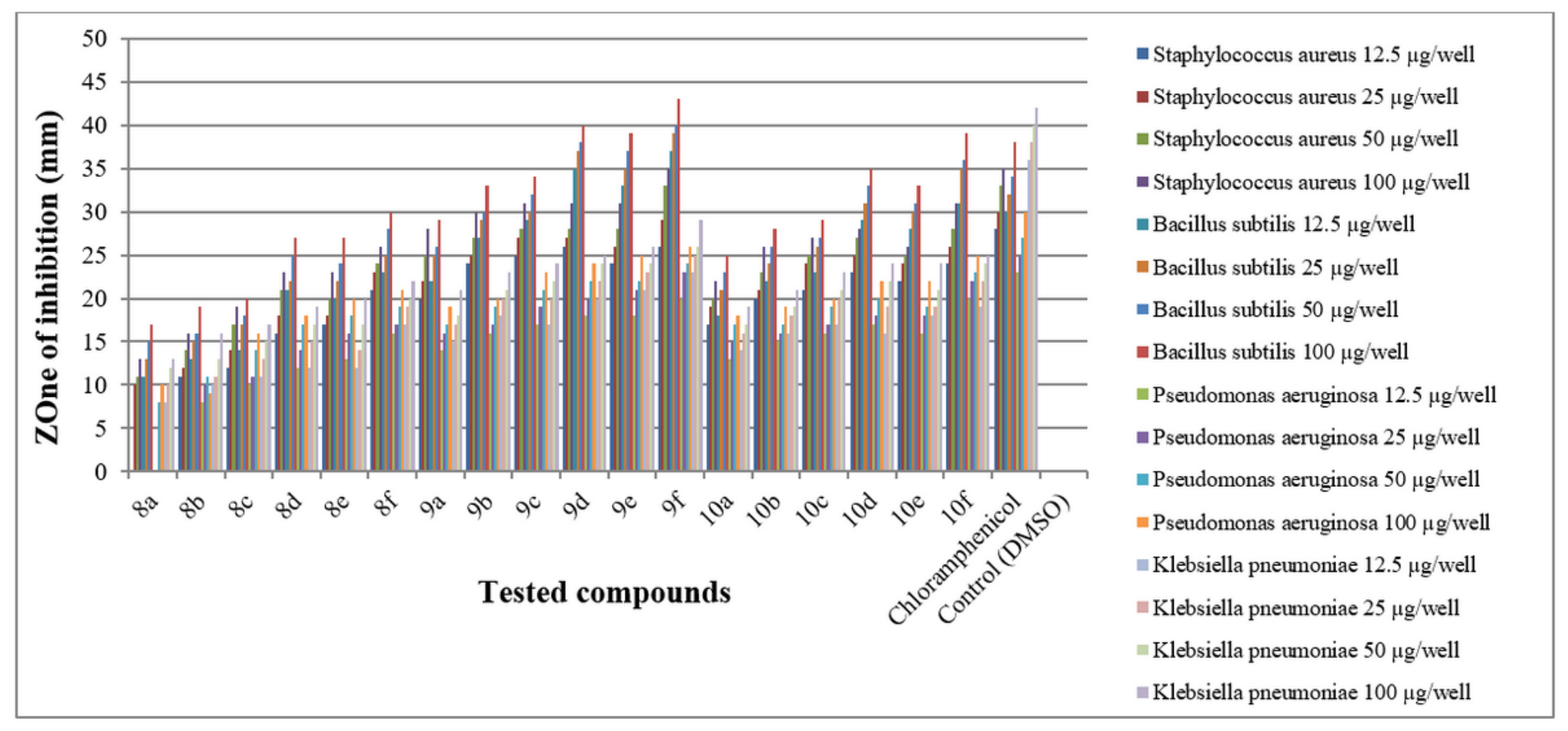

Figure 1

The invitro antibacterial activity of compounds 8-10 


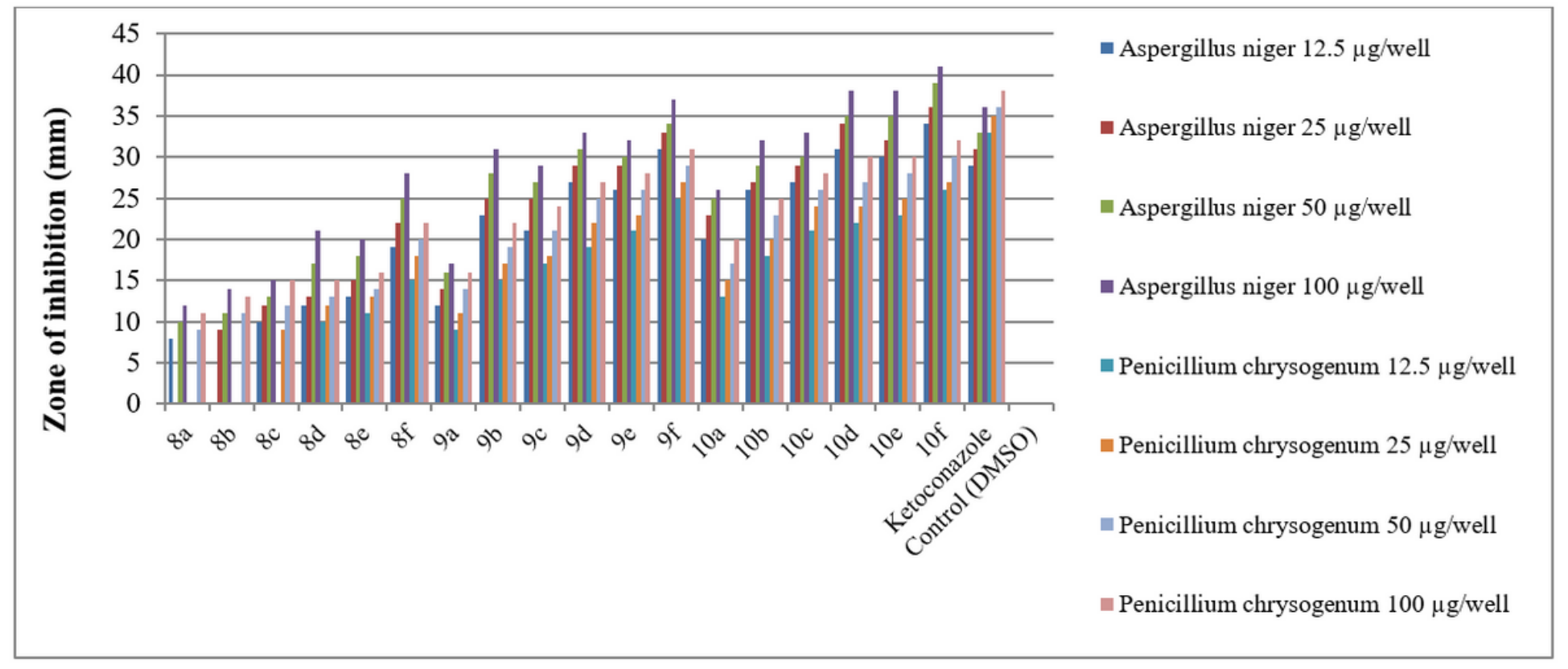

Figure 2

The in vitro antifungal activity of compounds 8-10

\section{Supplementary Files}

This is a list of supplementary files associated with this preprint. Click to download.

- Table2.docx

- Supplementaryinformation.docx

- Scheme1.png

- Scheme2.png 